\title{
Self-Enforcing Intergenerational Social Contract as a Source of Pareto Improvement and Pollution Mitigation
}

\author{
Nguyen Thang Dao \\ Kerstin Burghaus \\ Ottmar Edenhofer
}
CESIFO WORKING PAPER NO. 5896
CATEgORY 10: ENERGY AND ClimATE ECONOMICS
MAY 2016

An electronic version of the paper may be downloaded

- from the SSRN website:

- from the RePEc website:

- from the CESifo website:

www.SSRN.com

www.RePEc.org

www.CESifo-group.org/wp 


\title{
Self-Enforcing Intergenerational Social Contract as a Source of Pareto Improvement and Pollution Mitigation
}

\begin{abstract}
We contribute to the literature on private financing of intergenerational public goods, focusing on climate change mitigation. We consider, in a general equilibrium overlapping generations (OLG) model with environmental externalities, a contract between successive generations, whereby agents of the current working-age generation invest a share of their labor income in pollution mitigation in exchange for a transfer to their old-age capital income paid by the next generation. We show that the existence of a contract which is Pareto-improving compared to an equilibrium without contract requires a minimum level of income and we characterize the set of Pareto-improving mitigation-transfer combinations. Nash bargaining yields unique solutions for the mitigation share and transfer rate respectively which increase in income. We prove that Nash bargaining leads to a steady state with lower pollution stock and higher income compared to the steady state without a contract and we study transitional dynamics. Notably, simulation shows that delaying the implementation of a social contract for too long may have considerable welfare costs: Income inevitably falls below the threshold in finite time so that Pareto improving mitigation is no longer possible and the economy converges to a steady state with high pollution stock and low income. In the second part of the paper, we study a game theoretic setup with overlapping generations, taking into account that credibly committing to a contract might not be possible. We show that if transfers are granted as a subsidy to capital income, there exist mitigation transfer schemes which are both Pareto improving and give no generation an incentive to deviate from the contract even in the absence of population growth. Further, the complete crowding out of investment incurred with lump sum transfers is avoided in our setup.
\end{abstract}

JEL-Codes: D620, D640, E210, Q540.

Keywords: OLG models, mitigation, Pareto improvement, social contract.

Nguyen Thang Dao

Mercator Research Institute on Global

Commons and Climate Change (MCC)

Torgauer Str. $12-15$

Germany - 10829 Berlin

daonguyen@mcc-berlin.net
Kerstin Burghaus

Mercator Research Institute on Global

Commons and Climate Change (MCC)

Torgauer Str. $12-15$

Germany - 10829 Berlin

burghaus@mcc-berlin.net

Ottmar Edenhofer

Mercator Research Institute on Global Commons and Climate Change (MCC), Potsdam Institute for Climate Impact Research (PIK) \& TU Berlin / Germany

edenhofer@pik-potsdam.de 
April 29, 2016

We thank Niko Jaakkola for his very insightful comments and Claudine Chen for her supports in simulation. The comments from participants of several conferences and workshops in Helsinki, Dublin, Münster, Strasbourg, and Amsterdam are also gratefully appreciated. The scientific responsibility lies with the authors. 


\section{Introduction}

Protecting environmental stocks like the climate or a natural resource affects generations unequally. It requires action of current generations while benefits accrue largely to future generations. This makes it difficult for policymakers to make environmental policy measures appealing to their voters. Still, it has been pointed out e.g. by Bovenberg and Heijdra (1998) and Gerlagh and Keyzer (2001) that environmental protection can be achieved in a Paretoimproving way, making also those alive today better off. The reason is that the benefits from environmental protection can be distributed over current and future generations such that each generation enjoys higher welfare. ${ }^{4}$ Foley (2007) even argues that protection can be achieved without any loss of consumption for any generation. Bovenberg and Heijdra (1998) and Foley (2007) suggest taxes to deal with the environmental externality and debt policy for intergenerational redistribution, while Gerlagh and Keyzer (2001) propose to establish a trust fund, giving equal claims over a natural resource to all current and future generations.

We study instead a contract between successive generations, whereby agents of the current working-age generation privately invest a share of their labor income in pollution mitigation. In exchange, they expect to receive a transfer to their old-age capital income from the next generation when retired. For this purpose, we set up a dynamic general equilibrium OLGmodel with an environmental externality: The stock of pollutions in a period reduces next period's total factor productivity. We show that the existence of a contract which is Pareto improving compared to an equilibrium without contract requires a sufficiently high level of income and we characterize the set of Pareto-improving mitigation-transfer combinations. Nash bargaining yields unique solutions for the mitigation share and transfer rate respectively which increase in income. We prove that steady state stock of pollutions under the Nash bargaining outcome are lower and steady state incomes are higher than without a contract. This is not trivial result because in our model, the long run effects of a social contract on the environment are priory ambiguous. The reason is that mitigation has an ambiguous effect on income and thereby capital accumulation. Note that the effects of policies on capital accumulation are neglected in the related models by Gerlagh and Keyzer (2001) as well as Karp and Rezai (2014). Another important result of our model is that delaying the implementation of a social contract for too long may have considerable welfare costs: Simulation shows that income inevitably falls below the threshold in finite time so that Pareto improving mitigation is no longer possible and the economy converges to a steady state with high pollution stock and low income.

There is a sizable literature studying the role of government intervention in the market economy for eliminating the dynamic inefficiency of overlapping generation (OLG) economies

\footnotetext{
${ }^{4}$ Karp and Rezai (2014) argue that environmental protection may, through a positive effect on productivity, lead to an appreciation in the price of capital and thereby benefit current generations. The conclusion is similar: Through intergenerational redistribution, a Pareto improvement can be obtained.
} 
with environmental externalities. An incomplete list of papers includes John and Pecchenino (1994), Ono (1996), Jouvet et al. (2000), Gutierrez (2008), Goenka et al. (2012), Fodha and Seegmuller (2014), Dao and Davila (2014), Dao and Edenhofer (2014). Contrary to the aforementioned authors, we do not derive and analyze the first best intertemporal optimum in our paper. Determining an intertemporal optiumum requires weighing the welfare of different groups of people at different points in time. Further, implementing the socially optimal allocation may be difficult if not infeasible from a political economy perspective. Our aim is to propose a politically more feasible way of implementing environmental protection. Note that we do also not search for an intertemporally efficient outcome: While we could characterize the Pareto frontier between two successive generations, van Below, Dennig and Jaakkola (2015, mimeo) show that including further, more distant generations into the contract leads to a Pareto improvement.

There is however another question concerning the feasibility of implementing intergenerational contracts for mitigation: Even if a contract proposed by generations currently alive improves the welfare of all generations involved, future generations may still have an incentive to default on the provisions of the contract if given the chance. Talking in terms of our model, once a generation has invested in mitigation, the succeeding generation might be even better off if it does not pay the transfer to the old generation's capital income. Knowing that future generations might not want to fulfill their obligations from the contract and that policy makers might not be able or willing to enforce them, current generations will not be willing to offer a contract in the first place. Neither of the papers cited above takes this commitment problem into account.

In the last part of our paper, we explicitly consider commitment problems in a game theoretic setup. We characterize the conditions under which a contract scheme is a subgame perfect equilibrium of a repeated game with overlapping generations. Such a scheme is selfenforcing in the sense that no generation has an incentive to deviate. We study the existence of a set of incentive compatible combinations of mitigation share and transfer and we show under which condition there exist combinations of mitigation share and transfer, for which a series of contracts is simultaneously Pareto-improving and self-enforcing. For such combinations, institutions are needed to provide information, coordinate payments and surveil compliance with the contract but agents of each generation would in every period voluntarily vote to continue the contract. Rangel (2003), Cigno (1993, 2006a, 2006b) and Anderberg and Balestrino (2003) analyze the scope for providing a public good (education, environmental quality) by linking its provision to pay-as-you go transfers in a game theoretic setup with non-altruistic agents. They show that a necessary condition to sustain the provision of the public good is that the return to the compensating intergenerational transfer must be sufficiently larger than the return to private savings. With a constant pay-as-you-go transfer, sufficiently fast population growth is needed to satisfy this condition. Further, intergenerational transfers crowd out 
capital investment. A crucial difference of our model is that we assume transfers are paid from labor income and redistributed to the old as a subsidy on capital income rather than pay-asyou-go pensions. In our model, a mitigation-transfer scheme can be sustainable in the absence of population growth if the capital income share is sufficiently below the labor income share. Further, capital investement is to some extent complementary to the transfer scheme. Finally, while the game-theoretic models above typically consider a partial equilibrium or small open economy with fixed interest rate, we consider a general equilibrium setup.

While we apply our model to the problem of climate change mitigation, it is of more general interest for the private financing of other intergenerational public goods like health, education or infrastructure.

We describe the setup of the model in section 2. In Section 3 we explain our understanding of a social contract, study the existence of a set of Pareto-improving combinations of mitigation investment and transfer and characterize this set. We then prove in section 4 that Nash bargaining yields a unique solution for the mitigation share and transfer which is increasing in income. Further, we compare the dynamic behavior of the model without social contracts and with social contracts determined by Nash bargaining in each period. We prove that under period-by-period social contracts, there exists a steady-state with lower pollution stock and higher income compared to the steady-state which is reached without social contracts. Finally, in section 6 , we investigate the dynamic sustainability of a mitigation-transfer scheme when agents are allowed to deviate from a contract ex-post. Section 7 concludes.

\section{The model}

\subsection{Dynamics of pollution stock}

We assume that the dynamics of pollution stock is given by

$$
E_{t}=\bar{E}+(1-\delta)\left(E_{t-1}-\bar{E}\right)+P_{t}
$$

where $E_{t} \in \mathbb{R}$ is an index of the pollution stock, which measures the carbon concentration in the atmosphere, in period $t ; P_{t}$ is the pollution flow released in the atmosphere due to human economic activities (say production and mitigation) in period $t$ and $\bar{E}$ is the natural state of carbon concentration in the atmosphere, i.e. the state of the ecological system without any human activity. $\delta \in[0,1]$ is the decay rate of pollution stock which measures the convergence speed of the pollution stock to the natural state $\bar{E}$. For simplicity, we normalize $\bar{E}=0$. Therefore, we hereafter rewrite the dynamics of pollution stock as

$$
E_{t}=(1-\delta) E_{t-1}+P_{t}
$$

We assume that the pollution flow $P_{t}$ is determined by 


$$
P_{t}=\xi K_{t}-\gamma M_{t}
$$

where $\xi K_{t}$ is the pollution from capital $K_{t}$, and $\gamma M_{t}$ is pollution abatement resulting from the mitigation effort $M_{t} . \xi, \gamma>0$ are pollution and mitigation coefficients of physical capital and mitigation respectively. Without loss of generality, we normalize $\xi=1$. So hereafter, the dynamics of pollution stock is

$$
E_{t}=(1-\delta) E_{t-1}+K_{t}-\gamma M_{t}
$$

\subsection{Production}

Aggregate production is given by

$$
Y_{t}=z\left(E_{t-1}\right) F\left(K_{t}, L_{t}\right)
$$

where $z\left(E_{t-1}\right)$ is total factor productivity in period $t$ which depends on the pollution stock in the previous period. Note that last period's pollution stock affects current productivity, reflecting the long-run effect of pollution. For simplification and expositional purpose, in this paper we consider the following functional form of $z(E)$ :

$$
z(E)=\left\{\begin{array}{l}
A \mathrm{e}^{-E} \quad \text { if } E \geq 0 \\
A \mathrm{e}^{E} \quad \text { if } E<0
\end{array} ; A>0\right.
$$

The damage function $z(E)$ above tells us that total factor productivity gets maximal when the carbon concentration in the atmosphere is at the natural state. In the following, we consider a production function of the Cobb-Douglas type, so that $z\left(E_{t-1}\right) F\left(K_{t}, L_{t}\right)=$ $z\left(E_{t-1}\right) K_{t}^{\alpha} L_{t}^{1-\alpha}, \alpha \in(0,1)$. We normalize each period to be one unit of time, and we assume that the capital stock fully depreciates during each period. The returns to capital and labor in period $t$ are determined through profit maximization of the producing firms as follows

$$
\begin{gathered}
R_{t}=z\left(E_{t-1}\right) F_{K}\left(K_{t}, L_{t}\right)=z\left(E_{t-1}\right) \alpha k_{t}^{\alpha-1} \\
w_{t}=z\left(E_{t-1}\right) F_{L}\left(K_{t}, L_{t}\right)=z\left(E_{t-1}\right)(1-\alpha) k_{t}^{\alpha}
\end{gathered}
$$

where $k_{t}=K_{t} / L_{t}$.

\subsection{Agents and the intergenerational social contract}

For simplification, we assume that each agent has one parent and one offspring and lives for 
three periods $t-1, t$, and $t+1$. In the first period of life (childhood) $t-1$, a generation of identical agents are children who cannot make choices to influence their respective utility levels. In the second period of life (young age) $t$, they are identical adults and each of them is endowed with 1 unit of labor. They supply their labor endowments inelastically to the market to get the labor incomes. During this period they allocate their net income between young-age consumption $c_{t}^{y}$ and savings $k_{t+1}$. Savings are consumed in the third period of life (old age) so as to maximize lifetime utility. Hereafter, we denote by agent $t$ or/and generation $t$ the agent and/or generation entering working age in period $t$. At the beginning (end) of period $t$, this generation negotiates with the preceding (succeeding) generation for pollution mitigation and an intergenerational transfer and signs a contract. We do not assume altruism between generations, so that each generation seeks to maximize its own welfare. In particular, the negotiation at the beginning of each period $t+1$ is the following: The young generation at $t+1$ (i.e. generation $t+1$ ) offers the contemporary old generation (i.e. generation $t$ ) to sign a contract according to which each agent $t$ leave a portion $m_{t} \in[0,1)$ of his young-age income for mitigation. In exchange, each agent $t$ will receive a transfer at rate $\tau_{t+1}^{o} \geq 0$ to his gross capital income when old. Note that the case $\left(m_{t}, \tau_{t+1}^{o}\right)=(0,0)$ implies the contract between the young and old generations at the beginning of period $t+1$ is not signed.

\begin{tabular}{ccc}
$t$ & $t+1$ & $t+2$ \\
\hdashline$\left.m_{t-1}, \tau_{t}^{o}\right)$ & $\bullet$ & $\bullet$ \\
$\left(m_{t}, \tau_{t+1}^{o}\right)$ & $\left(m_{t+1}, \tau_{t+2}^{o}\right)$
\end{tabular}

Figure 1: Negotiation between the young and old generations at the beginning of each period

\section{A young agent's problem}

We consider the behavior of an agent $t$ in period $t$ under the intergenerational social contract $\left(m_{t}, \tau_{t+1}^{o}\right)$ at the beginning of period $t+1$ with the suceeding generation. His/her problem is

$$
\max _{c_{t}^{y}, k_{t+1}, c_{t+1}^{o}} \ln c_{t}^{y}+\beta \ln c_{t+1}^{o}
$$

subject to

$$
\begin{gathered}
c_{t}^{y}+k_{t+1} \leq I_{t}\left(1-m_{t}\right) \\
c_{t+1}^{o} \leq R_{t+1}^{e} k_{t+1}\left(1+\tau_{t+1}^{o}\right)
\end{gathered}
$$

given real income $I_{t}$, the perfectly foreseen return to capital $R_{t+1}^{e}=R_{t+1}$ and the social contract $\left(m_{t}, \tau_{t+1}^{o}\right) ; \beta \in(0,1)$ denotes the agent's time preference parameter.

The agent's optimal choices are 


$$
\begin{gathered}
c_{t}^{y}=\frac{1}{1+\beta} I_{t}\left(1-m_{t}\right) \\
k_{t+1}=\frac{\beta}{1+\beta} I_{t}\left(1-m_{t}\right) \\
c_{t+1}^{o}=\frac{R_{t+1} \beta}{1+\beta} I_{t}\left(1-m_{t}\right)\left(1+\tau_{t+1}^{o}\right)
\end{gathered}
$$

\subsection{Equilibrium}

Given an intergenerational social contract $\left(m_{t}, \tau_{t+1}^{o}\right)$, the competitive equilibrium in the economy is characterized by: (i) utility maximization of each agent (3) under the budget constraints (4) and (5); (ii) the law of motion of capital $K_{t+1}=k_{t+1}$; (iii) the maximization problem of the final good producing firm determining the returns of production factors (1) and (2); (iv) the dynamics of the pollution stock; and $(\mathrm{v})$ the balanced budget constraint of intergenerational transfers. Therefore, the set $\left\{c_{t}^{y}, k_{t+1}, c_{t+1}^{o}, E_{t}, P_{t}\right\}_{t}$, which fully characterizes the competitive equilibrium of the economy, is the solution to the following system of equations:

$$
\begin{gathered}
c_{t}^{y}=\frac{I_{t}\left(1-m_{t}\right)}{1+\beta} \\
k_{t+1}=\frac{\beta}{1+\beta} I_{t}\left(1-m_{t}\right) \\
c_{t+1}^{o}=\frac{z\left(E_{t}\right) F_{K}\left(k_{t+1}, 1\right) \beta}{1+\beta} I_{t}\left(1-m_{t}\right)\left(1+\tau_{t+1}^{o}\right) \\
E_{t}=(1-\delta) E_{t-1}+P_{t} \\
P_{t}=k_{t}-\gamma M_{t}
\end{gathered}
$$

given $k_{t}, E_{t-1}$, and $M_{t}$, where

$$
I_{t}=z\left(E_{t-1}\right)(1-\alpha) k_{t}^{\alpha}\left(1-\tau_{t}^{y}\right)
$$

is the income of agent $t$ after subtracting the transfer at rate $\tau_{t}^{y}$ to the contemporary old generation. $\tau_{t}^{y}$ and $\tau_{t}^{o}$ satisfy

$$
\tau_{t}^{y} z\left(E_{t-1}\right)(1-\alpha) k_{t}^{\alpha}=\tau_{t}^{o} z\left(E_{t-1}\right) \alpha k_{t}^{\alpha} ; \quad \text { i.e. } \quad \tau_{t}^{y}=\frac{\alpha}{1-\alpha} \tau_{t}^{o}
$$

which reflects the balanced intergenerational transfer constraint between generations $t$ and 
$t+1$.

Further, mitigation effort $M_{t}$ in period $t$ equals total mitigation investment $m_{t-1} I_{t-1}$ by generation $t-1$ :

$$
M_{t}=m_{t-1} I_{t-1}
$$

\section{Intergenerational social contract}

We now define the perfectly foreseen indirect utilities of agents at equilibrium and at the timing of negotiation for the intergenerational social contract. Since the damage effect is larger (i.e. lower $z\left(E_{t}\right)$ ) when the carbon concentration index is farther from the natural state $(E=0)$, rational agents will never reach a contract under which the carbon concetration is negative. Hence, hereafter, we always impose implicitly or explicitly a non-negativity contraint on the carbon concentration index:

$$
E_{t+1}=(1-\delta) E_{t}+\frac{\beta I_{t}\left(1-m_{t}\right)}{1+\beta}-\gamma m_{t} I_{t} \geq 0 \quad \forall t
$$

This constraint is equivalent to

$$
m_{t} \leq \frac{(1-\delta)(1+\beta) E_{t} / I_{t}+\beta}{\beta+\gamma+\gamma \beta} \equiv \bar{m}\left(I_{t}, E_{t}\right)=\bar{m}_{t} \quad \forall t
$$

\subsection{Agent $t$ at $t+1$}

The perfectly foreseen indirect utility of working-age agent $t$ under the contract $\left(m_{t}, \tau_{t+1}^{o}\right)$ with $m_{t} \leq \bar{m}_{t}$ at the beginning of time $t+1$ is

$$
\begin{gathered}
V_{t}^{t+1}\left(I_{t}, m_{t}, \tau_{t+1}^{o}\right)=\ln \left[\frac{1}{1+\beta} I_{t}\left(1-m_{t}\right)\right]+\beta \ln \left[\frac{R_{t+1} \beta}{1+\beta} I_{t}\left(1-m_{t}\right)\left(1+\tau_{t+1}^{o}\right)\right] \\
=\Phi+\beta \ln \left[z\left(E_{t}\right) \alpha k_{t+1}^{\alpha-1}\right]+(1+\beta)\left[\ln I_{t}+\ln \left(1-m_{t}\right)\right]+\beta \ln \left(1+\tau_{t+1}^{o}\right)
\end{gathered}
$$

where $\Phi=\ln \frac{1}{1+\beta}+\beta \ln \frac{\beta}{1+\beta}$.

We can also derive indirect utility of this agent without a social contract, which is

$$
V_{t}^{t+1}\left(I_{t}, 0,0\right)=\Phi+\beta \ln \left[z\left(E_{t}\right) \alpha \tilde{k}_{t+1}^{\alpha-1}\right]+(1+\beta) \ln I_{t}
$$

where $\tilde{k}_{t+1}$ is capital per capita if there is no social contract. From equation (10), we find that $\tilde{k}_{t+1}=\frac{\beta}{1+\beta} I_{t}=\frac{k_{t+1}}{1-m_{t}}$.

So, from (16) and (17), the foreseen surplus that agent $t$ gains from the social contract $\left(m_{t}, \tau_{t+1}^{o}\right)$ is 


$$
\triangle V_{t}^{t+1}=V_{t}^{t+1}\left(I_{t}, m_{t}, \tau_{t+1}^{o}\right)-V_{t}^{t+1}\left(I_{t}, 0,0\right)=\beta \ln \frac{k_{t+1}^{\alpha-1}}{\tilde{k}_{t+1}^{\alpha-1}}+(1+\beta) \ln \left(1-m_{t}\right)+\beta \ln \left(1+\tau_{t+1}^{o}\right)
$$

Knowing that $\tilde{k}_{t+1}=\frac{k_{t+1}}{1-m_{t}}$ we can rewrite the difference to be

$$
\triangle V_{t}^{t+1}=(1+\alpha \beta) \ln \left(1-m_{t}\right)+\beta \ln \left(1+\tau_{t+1}^{o}\right)
$$

We say the agent $t$ does not suffer a welfare-loss from the social contract $\left(m_{t}, \tau_{t+1}^{o}\right)$ if $\triangle V_{t}^{t+1} \geq 0$

\subsection{Agent $t+1$ at $t+1$}

The perfectly foreseen indirect utility of working-age generation $t+1$ under the contract $\left(m_{t}, \tau_{t+1}^{o}\right)$ with $m_{t} \leq \bar{m}\left(I_{t}, E_{t}\right)$ at the beginning of time $t+1$ is

$$
\begin{aligned}
\mathcal{V}_{t+1}^{t+1}\left(I_{t+1}, m_{t+1}^{e}, \tau_{t+2}^{o, e}\right)=\Phi & +\beta \ln \left[z\left(E_{t+1}\right) \alpha\left(\frac{\beta I_{t+1}\left(1-m_{t+1}^{e}\right)}{1+\beta}\right)^{\alpha-1}\right] \\
& +(1+\beta)\left[\ln I_{t+1}+\ln \left(1-m_{t+1}^{e}\right)\right]+\beta \ln \left(1+\tau_{t+2}^{o, e}\right)
\end{aligned}
$$

where $\left(m_{t+1}^{e}, \tau_{t+2}^{o, e}\right)$ is the foreseen social contract that the young generation $t+1$ will sign with the succeeding young generation $t+2$ at the beginning of period $t+2$, and $I_{t+1}=$ $z\left(E_{t}\right)(1-\alpha) k_{t+1}^{\alpha}\left(1-\tau_{t+1}^{y}\right)$ is the income of the young agent $t+1$ in period $t+1$ after paying the intergenerational transfer $\tau_{t+1}^{o} R_{t+1} k_{t+1}=z\left(E_{t}\right)(1-\alpha) k_{t+1}^{\alpha} \tau_{t+1}^{y}$ under the social contract $\left(m_{t}, \tau_{t+1}^{o}\right)$.

The perfectly foreseen indirect utility of working-age generation $t+1$ without the contract $\left(m_{t}, \tau_{t+1}^{o}\right)$ at the beginning of time $t+1$ is

$$
\begin{aligned}
\tilde{\mathcal{V}}_{t+1}^{t+1}\left(\tilde{I}_{t+1}, m_{t+1}^{e}, \tau_{t+2}^{o, e}\right)=\Phi & +\beta \ln \left[z\left(\tilde{E}_{t+1}\right) \alpha\left(\frac{\beta \tilde{I}_{t+1}\left(1-m_{t+1}^{e}\right)}{1+\beta}\right)^{\alpha-1}\right] \\
& +(1+\beta)\left[\ln \tilde{I}_{t+1}+\ln \left(1-m_{t+1}^{e}\right)\right]+\beta \ln \left(1+\tau_{t+2}^{o, e}\right)
\end{aligned}
$$

where $\tilde{I}_{t+1}=z\left(E_{t}\right)(1-\alpha) \tilde{k}_{t+1}^{\alpha}$ and $\tilde{E}_{t+1}$ are the income of the young agent $t+1$ and the stock of pollution, respectively, in period $t+1$ in the case of no contract $\left(m_{t}, \tau_{t+1}^{o}\right)$ with the preceding generation at the beginning of period $t+1$.

So, from (20) and (21), the foreseen surplus that the agent $t+1$ gains from the social contract $\left(m_{t}, \tau_{t+1}^{o}\right)$ is 


$$
\Delta \mathcal{V}_{t+1}^{t+1}=\mathcal{V}_{t+1}^{t+1}\left(I_{t+1}, m_{t+1}^{e}, \tau_{t+2}^{o, e}\right)-\tilde{\mathcal{V}}_{t+1}^{t+1}\left(\tilde{I}_{t+1}, m_{t+1}^{e}, \tau_{t+2}^{o, e}\right)=(1+\alpha \beta) \ln \frac{I_{t+1}}{\tilde{I}_{t+1}}+\beta \ln \frac{z\left(E_{t+1}\right)}{z\left(\tilde{E}_{t+1}\right)}
$$

We know that

$$
\frac{I_{t+1}}{\tilde{I}_{t+1}}=\frac{k_{t+1}^{\alpha}\left(1-\tau_{t+1}^{y}\right)}{\tilde{k}_{t+1}^{\alpha}}
$$

where $k_{t+1}=\tilde{k}_{t+1}\left(1-m_{t}\right)=\frac{\beta I_{t}}{1+\beta}\left(1-m_{t}\right), \tilde{P}_{t+1}=\tilde{k}_{t+1}, P_{t+1}=k_{t+1}-\gamma m_{t} I_{t}$, and $\tau_{t+1}^{y}=\frac{\alpha}{1-\alpha} \tau_{t+1}^{o}$. Therefore,

$$
\frac{I_{t+1}}{\tilde{I}_{t+1}}=\left(1-\frac{\alpha \tau_{t+1}^{o}}{1-\alpha}\right)\left(1-m_{t}\right)^{\alpha}
$$

We also know that

$$
\frac{z\left(E_{t+1}\right)}{z\left(\tilde{E}_{t+1}\right)}=\mathrm{e}^{\tilde{E}_{t+1}-E_{t+1}}=\mathrm{e}^{\tilde{P}_{t+1}-P_{t+1}}=\mathrm{e}^{\frac{\beta+\gamma+\gamma \beta}{1+\beta} m_{t} I_{t}}
$$

We say that agent $t+1$ does not suffer a welfare-loss from the social contract $\left(m_{t}, \tau_{t+1}^{o}\right)$ if

$$
\triangle \mathcal{V}_{t+1}^{t+1}=(1+\alpha \beta) \ln \left[\left(\frac{1-\alpha\left(1+\tau_{t+1}^{o}\right)}{1-\alpha}\right)\left(1-m_{t}\right)^{\alpha}\right]+\frac{\beta(\beta+\gamma+\gamma \beta)}{1+\beta} m_{t} I_{t} \geq 0
$$

\subsection{Existence of a Pareto-improving intergenerational social contract}

We consider a social contract $\left(m_{t}, \tau_{t+1}^{o}\right)$ a Pareto improvement over the situation without contract if under the contract, both generations $t$ and $t+1$ are better off. In this subsection, we will derive a condition that guarantees the existence of such a social contract. We define the set $\mathcal{S}_{t+1}^{o}$ such that

$$
\mathcal{S}_{t+1}^{o}=\left\{\left(m_{t}, \tau_{t+1}^{o}\right) \in\left[0, \bar{m}\left(I_{t}, E_{t}\right)\right] \times \mathbb{R}, m_{t}<1: \triangle V_{t}^{t+1}>0\right\}
$$

i.e. the set consists of all pairs $\left(m_{t}, \tau_{t+1}^{o}\right)$ which make generation $t$ better off. Further, we define the boundary of $\mathcal{S}_{t+1}^{o}$ as

$$
\underline{\mathcal{S}}_{t+1}^{o}=\left\{\left(m_{t}, \tau_{t+1}^{o}\right) \in\left[0, \min \left\{1, \bar{m}_{t}\right\}\right] \times \mathbb{R}, m_{t}<1: \triangle V_{t}^{t+1}=0\right\}
$$

which can be represented by the function

$$
\tau_{t+1}^{o}=\left(\frac{1}{1-m_{t}}\right)^{\frac{1}{\beta}+\alpha}-1 \equiv \Omega\left(m_{t}\right) \quad \text { with } \quad m_{t} \in\left[0, \min \left\{1, \bar{m}_{t}\right\}\right], m_{t}<1
$$

$\Omega\left(m_{t}\right)$ shows all pairs $\left(m_{t}, \tau_{t+1}^{o}\right)$ which leave generation $t$ just as well off with the contract. 
And we define the set $\mathcal{S}_{t+1}^{y}$ such that

$$
\mathcal{S}_{t+1}^{y}=\left\{\left(m_{t}, \tau_{t+1}^{o}\right) \in\left[0, \bar{m}\left(I_{t}, E_{t}\right)\right] \times \mathbb{R}, m_{t}<1: \Delta \mathcal{V}_{t+1}^{t+1}>0\right\}
$$

All pairs $\left(m_{t}, \tau_{t+1}^{o}\right) \in \mathcal{S}_{t+1}^{y}$ increase welfare of generation $t+1$. We define the boundary of $\mathcal{S}_{t+1}^{y}$, that is all pairs which leave generation $t+1$ just as well off with the contract as without, as

$$
\underline{\mathcal{S}}_{t+1}^{y}=\left\{\left(m_{t}, \tau_{t+1}^{o}\right) \in\left[0, \min \left\{1, \bar{m}_{t}\right\}\right] \times \mathbb{R}, m_{t}<1: \Delta \mathcal{V}_{t+1}^{t+1}=0\right\}
$$

which we can also express as

$\tau_{t+1}^{o}=\frac{1-\alpha}{\alpha}\left[1-\mathrm{e}^{-\frac{(\beta+\gamma+\gamma \beta) \beta m_{t} I_{t}}{(1+\beta)(1+\alpha \beta)}}\left(1-m_{t}\right)^{-\alpha}\right] \equiv \psi\left(m_{t}, I_{t}\right) \quad$ with $\quad m_{t} \in\left[0, \min \left\{1, \bar{m}_{t}\right\}\right], m_{t}<1$

Lemma 1. The sets $\mathcal{S}_{t+1}^{o}$ and $\mathcal{S}_{t+1}^{y}$ are convex.

Proof. See Appendix A1.

Define

$$
\mathcal{P}_{t+1}^{t}=\mathcal{S}_{t+1}^{o} \cap \mathcal{S}_{t+1}^{y}
$$

The set $\mathcal{P}_{t+1}^{t}$ is the Pareto improvement set between generations $t$ and $t+1$. It contains any pair $\left(m_{t}, \tau_{t+1}^{o}\right) \in \mathcal{P}_{t+1}^{t}$ which makes both generations $t$ and $t+1$ better off compared to the pair $\left(m_{t}, \tau_{t+1}^{o}\right)=(0,0)$. The condition for the nonemptiness of this set is stated in proposition 1 below.

Proposition 1. In the economy set up above, there exists a threshold $\hat{I}$ of real income such that $\mathcal{P}_{t+1}^{t} \neq \oslash$ if, and only if the income of agent $t$ exceeds that threshold. i.e. $I_{t}>\hat{I}$.

Proof. See Appendix A2.

Proposition 1 states the condition on real income of agent $t$ under which a set of Paretoimproving pairs $\left(m_{t}, \tau_{t+1}^{o}\right)$ exists. We show in the appendix that the income threshold is given by

$$
\hat{I}=\frac{\alpha(1+\alpha \beta)(1+\beta)^{2}}{(1-\alpha)(\beta+\gamma+\gamma \beta) \beta^{2}}
$$

By taking the derivatives, it can be shown that $\hat{I}$ increases in the capital share $\alpha$ and decreases in the rate of time preference $\beta$ and the efficiency of the mitigation technology as given by $\gamma$. 
We will now provide some intuition for the presence of an income threshold and its dependency on parameters. Note that the existence of a Pareto-improving set of pairs $\left(m_{t}, \tau_{t+1}^{o}\right)$ depends on the slopes of $\Omega\left(m_{t}\right)$ and $\psi\left(m_{t}, I_{t}\right)$ at the origin in the $\left(m_{t}, \tau_{t+1}^{o}\right)$-plane. ${ }^{5}$ These slopes implicitly reflect the marginal utility gains and losses of the old and the young generation from engaging in an intergenerational social contract. For the old generation, generation $t$, the higher $\Omega^{\prime}\left(0^{+}\right)$, the larger the minimum increase in the transfer which is required to make generation $t$ just as well off with the contract as without it. As can be seen in the appendix, $\Omega^{\prime}\left(0^{+}\right)$is larger when the net marginal utility loss $1+\alpha \beta$ from an increase in mitigation is large compared to $\beta$, the marginal utility gain from an increase in the transfer $\tau_{t+1}^{o}$ received in the second period of life. The net marginal utility loss results from the reduction in disposable income due to mitigation investment $m_{t}$ and the associated decline in savings which is only partly compensated by an increase in the equilibrium interest rate $R_{t+1}$.

For the young generation, generation $t+1$, the higher $\psi_{m}\left(0^{+}, I_{t}\right)$, the higher the marginal increase in the transfer $\tau_{t+1}^{o}$ they are able to pay in return for a larger mitigation share $m_{t}$ without incurring a welfare loss. Generation $t+1$ suffers from a reduction in working-age income, both directly because it has to pay the transfer to the old, and indirectly because the old accumulate less capital under the contract. The marginal utility effect of this income reduction is again $1+\alpha \beta$. On the other hand, the mitigation share left by old leads to less pollution which has a positive productivity effect and increases the expected returns to savings. This latter effect is stronger when more weight is put on the second period of life (higher $\beta$ ), when the efficiency of the mitigation technology as measured by $\gamma$ is greater and when income $I_{t}$ is larger. The higher $\gamma$ and/or income, the greater the reduction in the pollution stock for a given income share invested in mitigation, $m_{t}$.

So, for an intergenerational social contract to exist, it is important that $\psi_{m}\left(0^{+}, I_{t}\right)>\Omega^{\prime}\left(0^{+}\right)$: The marginal increase in the transfer $\tau_{t+1}^{o}$ the young generation $t+1$ can accept must exceed the minimum increase the old generation $t$ requires as a compensation for leaving a share $m_{t}$ of its income for mitigation. The existence and nonexistence of $\mathcal{P}_{t+1}^{t}$ are depicted in figure 2.

Lemma 2. $\mathcal{P}_{t+1}^{t} \cup \underline{\mathcal{P}}_{t+1}^{t}$ constitutes a compact set, where $\underline{\mathcal{P}}_{t+1}^{t}$ is the boundary of $\mathcal{P}_{t+1}^{t}$.

Proof. See Appendix A3.

Note that the compactness of $\mathcal{P}_{t+1}^{t} \cup \underline{\mathcal{P}}_{t+1}^{t}$ stated in lemma 2 is important in guaranteeing the existence of a Nash bargaining solution, which is introduced in the next section, between generations.

\footnotetext{
${ }^{5}$ The slopes are given in appendix A2. but can also be easily derived from equations (25) and (27).
} 

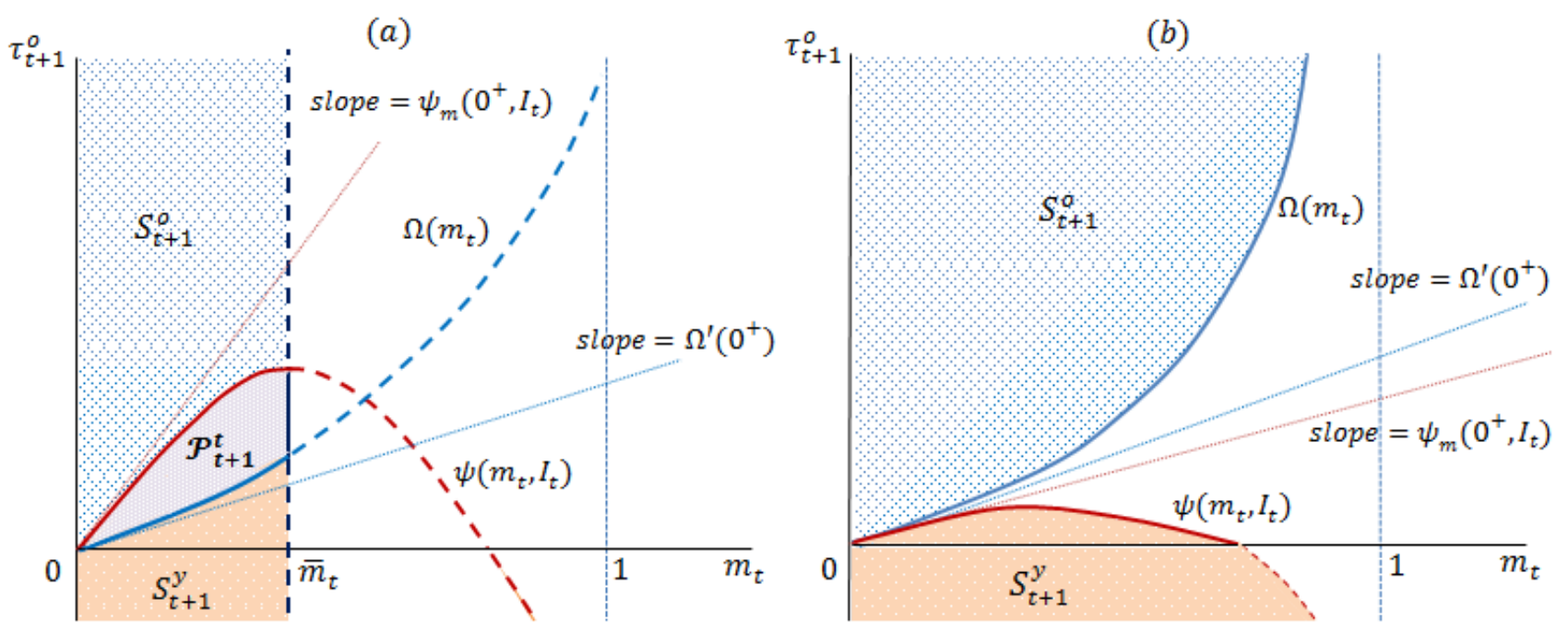

Figure 2: The existence and nonexistence of $\mathcal{P}_{t+1}^{t}:(a)$ the case $I_{t}>\hat{I}$; and $(b)$ the case $I_{t} \leq \hat{I}$.

\section{Bargaining problem, dynamics and steady state}

\subsection{Bargaining problem}

Indeed, any pair $\left(m_{t}, \tau_{t+1}^{o}\right) \in \mathcal{P}_{t+1}^{t}$ makes both generations $t$ and $t+1$ better off and even if we focus on Pareto efficiency, there are infinitely many Pareto efficient combinations of $m_{t}$ and $\tau_{t+1}^{o}$. Which $\left(m_{t}, \tau_{t+1}^{o}\right) \in \mathcal{P}_{t+1}^{t}$ will be the outcome of the negotiations between generations? The agreement $\left(m_{t}, \tau_{t+1}^{o}\right)$ in general depends on the bargaining power of each generation. Unfortunately, the bargaining theory has so far said relatively little about the determinant of bargaining power. In the following, we will consider the so-called Nash bargaining problem. In his seminal paper, Nash (1950) introduced a two-person bargaining theory and provided an normative approach to finding a solution to the Nash bargaining problem. Note that the Nash bargaining solution is Pareto efficient. In our context, the Nash solution $\left(m_{t}^{*}, \tau_{t+1}^{o *}\right)$, given it exists, is

$$
\left(m_{t}^{*}, \tau_{t+1}^{o *}\right) \in \underset{\left(m_{t}, \tau_{t+1}^{o}\right) \in \mathcal{P}_{t+1}^{t} \cup \underline{\mathcal{P}}_{t+1}^{t}}{\arg \max }\left\{\triangle V_{t}^{t+1} \triangle \mathcal{V}_{t+1}^{t+1}\right\}
$$

Proposition 2. For all $I_{t}>\hat{I}$, then there exists a unique point $\left(m_{t}^{*}, \tau_{t+1}^{o *}\right) \in \mathcal{P}_{t+1}^{t}$ solving the Nash bargaining problem (29). If $\left(m_{t}^{*}, \tau_{t+1}^{o *}\right)$ is an interior point in $\mathcal{P}_{t+1}^{t}$, then it is the unique stationary point for the function $\triangle V_{t}^{t+1} \triangle \mathcal{V}_{t+1}^{t+1}$ defined over the set $\mathcal{P}_{t+1}^{t} \cup \underline{\mathcal{P}}_{t+1}^{t}{ }^{6}$

Proof. See Appendix A4.

It is interesting to find a condition under which the constraint $m_{t} \leq \bar{m}_{t}$ is binding. In other

\footnotetext{
${ }^{6}$ The uniqueness of the solution and the stationary point is important because it helps us to rule out the case of bifurcation when we study the dynamic system.
} 
word, we wish to find a condition under which

$$
m_{t}^{*}=\frac{(1-\delta)(1+\beta) E_{t}+\beta I_{t}}{(\beta+\gamma+\gamma \beta) I_{t}} \equiv \bar{m}_{t}
$$

where $m_{t}^{*}=\underset{m_{t}}{\arg \max } \Delta V_{t}^{t+1} \triangle \mathcal{V}_{t+1}^{t+1}$ subject to $\Delta V_{t}^{t+1} \geq 0$ and $\Delta \mathcal{V}_{t+1}^{t+1} \geq 0 .{ }^{7}$ This condition allows us to characterize an area such that when the economy enters this area, the stock of pollution will converge to the natural state, i.e. $E=0$. We call this area the "Ideal area". Because such an area is not a focus of our paper and for simplification, without substantial loss of generality, we consider the area under the following assumption

Assumption 1. $\frac{\beta+\gamma+\gamma \beta}{\alpha(1+\beta)}\left[\frac{\beta(1-\alpha)}{1+\beta}\right]^{\frac{2-\alpha}{1-\alpha}}<1$.

This assumption is a sufficient condition to guarantee that $m_{t}^{*}$ in (30) is increasing in $I_{t}$. This assumption can hold in practice when the mitigation efficiency parameter $\gamma$ is not too high. Note that in this set up we normalize the pollution effect of capital to 1 , so it is plausible that the mitigation efficiency parameter is around this value. Assumption 1 holds when we set plausible values $\alpha=0.3, \beta=0.6$ and $\gamma=1$.

Similar to the proof of Proposition 2 this optimization problem has a unique interior solution and it holds that

$$
\begin{aligned}
\hat{Q}\left(m_{t}^{*} ; I_{t}\right)=\ln & {\left[\left(1-m_{t}^{*}\right)^{1+\alpha \beta}\left(\frac{\beta b I_{t}\left(1-m_{t}^{*}\right)-\alpha \beta(1+\alpha \beta)}{\alpha\left(\beta b I_{t}\left(1-m_{t}^{*}\right)+1+\alpha \beta\right)}\right)^{\beta}\right]^{b I_{t}-\frac{\alpha(1+\alpha \beta)}{1-m_{t}^{*}}} } \\
& -\frac{1+\alpha \beta}{1-m_{t}^{*}}\left\{\ln \left[\frac{\left(1-\alpha^{2} \beta^{2}\right)\left(1-m_{t}^{*}\right)^{\alpha}}{(1-\alpha)\left(\beta b I_{t}\left(1-m_{t}^{*}\right)+1+\alpha \beta\right)}\right]^{1+\alpha \beta}+b m_{t}^{*} I_{t}\right\}=0
\end{aligned}
$$

where $\hat{Q}_{m}\left(m_{t}^{*} ; I_{t}\right)<0$ and, under assumption $1, \hat{Q}_{I}\left(m_{t}^{*} ; I_{t}\right)>0$ for all $I_{t} \in(\hat{I}, \bar{I})$, in which $\bar{I}=[A(1-\alpha)]^{\frac{1}{1-\alpha}}\left(\frac{\beta}{1+\beta}\right)^{\frac{\alpha}{1-\alpha}}$ is conditional steady state income in the case of no social contract and pollution stock is set at $E=0$. By applying the implicit function theorem we have

$$
\forall I_{t} \in(\hat{I}, \bar{I}), \quad m_{t}^{*}=m\left(I_{t}\right) \quad \text { and } \quad m^{\prime}\left(I_{t}\right)>0
$$

Lemma 3. If $m(\bar{I})>\frac{\beta}{\beta+\gamma+\gamma \beta}$, then there exists $\underline{I} \in(\hat{I}, \bar{I})$ and $\hat{E}_{t}=\hat{E}\left(I_{t}\right)$ for $I_{t} \in(\underline{I}, \bar{I})$ such that $m_{t}^{*}=\bar{m}_{t}$ (or equivalently $E_{t+1}=0$ ) if, and only if $E_{t} \leq \hat{E}\left(I_{t}\right)$. Moreover, $\hat{E}(\underline{I})=0$, and $\hat{E}^{\prime}\left(I_{t}\right)>0$ for $I_{t} \in[\underline{I}, \bar{I}]$.

Proof. See Appendix A5.

\footnotetext{
${ }^{7}$ Note that in this optimization we ignore the contraint $m_{t} \leq \bar{m}_{t}$ in order to find the condition under which this constraint is just binding. And note also that we substitute $\tau_{t+1}^{o}$ as a function of $m_{t}$, as derived from the proof of proposition 2 in the Appendix A4.
} 
Lemma 3 reveals the existence of an area in the North-East of the $I-E$ plane such that under period-by-period social contracts an economy starting from any point in this area may converge to a steady state with $E_{*}=0$ and $I_{*} \in(\underline{I}, \bar{I})$, that is, a steady state in the ideal area. The proof for existence of this steady state is fairly straightforward because starting from any point $\left(I_{t}, E_{t}\right)$ in the ideal area leads to a social contract with $m_{t}=\frac{(1-\delta)(1+\beta) E_{t}+\beta I_{t}}{(\beta+\gamma+\gamma \beta) I_{t}}$. Hence, from $t+1$ onwards $E=0$ and $m=\frac{\beta}{\beta+\gamma+\gamma \beta}$. The existence and uniqueness of the transfer $\tau_{t+1}^{o *}$ is proved in Proposition 2. Since $I_{t+1}=z\left(E_{t}\right)\left(\frac{\beta\left(1-m_{t}\right) I_{t}}{1+\beta}\right)^{\alpha}\left[1-\alpha\left(1+\tau_{t+1}^{o}\right)\right]$, the steady state is characterized by

$$
I_{*}^{1-\alpha}-A\left(\frac{\gamma \beta}{\beta+\gamma+\gamma \beta}\right)^{\alpha}\left[1-\alpha\left(1+\tau\left(I_{*}\right)\right)\right]=0
$$

which always guarantees the existence and uniqueness of the steay state.

\subsection{Dynamics and steady states}

We first consider the steady state of the economy in the case of no social contract. With $\left(m_{t}, \tau_{t+1}^{o}\right)=(0,0)$ for all $t$, the dynamic system is fully characterized by the evolution of real income and stock of pollution as follows

$$
\begin{gathered}
I_{t+1}=A \mathrm{e}^{-E_{t}}\left[\frac{\beta I_{t}}{1+\beta}\right]^{\alpha}(1-\alpha) \\
E_{t+1}=(1-\delta) E_{t}+\frac{\beta}{1+\beta} I_{t}
\end{gathered}
$$

Proposition 3. Without intergenerational social contracts in all periods the economy converges to a globally stable steady state.

Proof. See Appendix A6.

We next study the dynamic system with the social contract under the Nash bargaining problem. The dynamic system is now characterized by the following equations

$$
\begin{gathered}
I_{t+1}=A \mathrm{e}^{-E_{t}}\left[\frac{\beta I_{t}\left(1-m_{t}^{*}\right)}{1+\beta}\right]^{\alpha}\left[1-\alpha\left(1+\tau_{t+1}^{o *}\right)\right] \\
E_{t+1}=(1-\delta) E_{t}+\frac{\beta-(\beta+\gamma+\gamma \beta) m_{t}^{*}}{1+\beta} I_{t}
\end{gathered}
$$

given $I_{0}, E_{0}>0$. Note that for the case $I_{t} \leq \hat{I}$, we set $\left(m_{t}^{*}, \tau_{t+1}^{o *}\right)=(0,0)$.

We now consider the dynamics, characterized by the system (33)-(34), and the steady states of the economy under the intergenerational social contract $\left(m_{t}^{*}, \tau_{t+1}^{o *}\right)$. In order to guarantee the 
existence of a steady state with intergenerational social contracts, it is necessary to introduce the following assumption

Assumption 2. $\bar{I}>\hat{I}$, i.e. $A>\left[\frac{\alpha(1+\alpha \beta)}{\beta+\gamma+\gamma \beta}\right]^{1-\alpha}\left[\frac{1+\beta}{\beta(1-\alpha)}\right]^{2-\alpha}$.

This assumption implies that the economy's level of technology $A$ should be sufficiently high. Otherwise the economy always converges to the steady state $(\tilde{I}, \tilde{E})$, i.e. the steady state without social contract.

The first property of the steady state under the intergenerational social contract $\left(m_{t}^{*}, \tau_{t+1}^{o *}\right)$ is stated in the next proposition

Proposition 4. Under the intergenerational social contracts $\left(m_{t}^{*}, \tau_{t+1}^{o *}\right) \in \mathcal{P}_{t+1}^{t}$, when $I_{t}>\hat{I}$, and if $E E\left(m_{t}^{*}, \tau_{t+1}^{o *}\right) \cap I I\left(m_{t}^{*}, \tau_{t+1}^{o *}\right) \neq \oslash$, the pollution stock at a steady state with social contract is lower than that at the steady state without social contract.

Proof. From the dynamic system (33)-(34), a steady state with social contract is characterized by

$$
\begin{gathered}
I^{1-\alpha}=A \mathrm{e}^{-E}\left[\frac{\beta(1-m)}{1+\beta}\right]^{\alpha}[1-\alpha(1+\tau)]>\hat{I}^{1-\alpha} \\
\delta E=\frac{\beta-(\beta+\gamma+\gamma \beta) m}{1+\beta} I \geq 0
\end{gathered}
$$

where $(m, \tau) \in \mathcal{P} \neq \oslash$ is the social contract at the steady state. Since $\hat{I}>0$, it follows from two last equations that $\tau<\frac{1-\alpha}{\alpha}$ and $m \leq \frac{\beta}{\beta+\gamma+\gamma \beta}$.

The stock of pollution at the steady state with social contracts is characterized by

$$
\mathrm{e}^{\frac{E}{1-\alpha}} E=A^{\frac{1}{1-\alpha}} \frac{\beta-(\beta+\gamma+\gamma \beta) m}{\delta(1+\beta)}\left[\frac{\beta(1-m)}{1+\beta}\right]^{\frac{\alpha}{1-\alpha}}[1-\alpha(1+\tau)]^{\frac{1}{1-\alpha}}
$$

It is straightforward from (35) that $E$ is decreasing in both $m$ and $\tau$ so that

$$
E=E(m, \tau)<E(0,0)=\tilde{E}
$$

Now it would be interesting to illustrate dynamics and convergence to the steady states intuitively. We focus on the most interesting dynamics which will be supported by our simulations in the later section. We study two distinct cases, $\hat{I}<\tilde{I}$ and $\hat{I}>\tilde{I}$, and present the dynamics through the phase diagrams. Define the sets $I I$ and $E E$ corresponding to the dynamic system given by (33) and (34) as follows

$$
I I \equiv\left\{\left(I_{t}, E_{t}\right) \in \Re_{+}^{2}: I_{t+1}=I_{t}\right\}
$$


i.e. $\quad E_{t}=\ln A+\alpha \ln \left[\frac{\beta\left(1-m\left(I_{t}\right)\right)}{1+\beta}\right]+\ln \left[1-\alpha\left(1+\tau\left(I_{t}\right)\right)\right]-(1-\alpha) \ln I_{t} \equiv \Gamma\left(I_{t}\right)$

and

$$
\begin{gathered}
E E \equiv\left\{\left(I_{t}, E_{t}\right) \in \Re_{+}^{2}: E_{t+1}=E_{t}\right\} \\
\text { i.e. } \quad E_{t}=\frac{\beta-(\beta+\gamma+\gamma \beta) m\left(I_{t}\right)}{\delta(1+\beta)} I_{t} \equiv \Lambda\left(I_{t}\right)
\end{gathered}
$$

Lemma 4. For the dynamic system $\left(I_{t}, E_{t}\right)_{t}$ characterized by equations (33)-(34), it holds that:

(i) $I_{t+1}-I_{t}\left\{\begin{array}{ccc}>0 & \text { if } & 0<E_{t}<\Gamma\left(I_{t}\right) \\ =0 & \text { if } \quad E_{t}=\Gamma\left(I_{t}\right) \\ <0 & \text { if } & E_{t}>\Gamma\left(I_{t}\right)\end{array} \quad\right.$ and $\quad$ (ii) $\quad E_{t+1}-E_{t}\left\{\begin{array}{ccc}>0 & \text { if } & 0<E_{t}<\Lambda\left(I_{t}\right) \\ =0 & \text { if } & E_{t}=\Lambda\left(I_{t}\right) \\ <0 & \text { if } & E_{t}>\Lambda\left(I_{t}\right)\end{array}\right.$

Proof. The proof for this Lemma is fairly straightforward.

In the following phase diagrams, the dashed lines $I I(0,0)$ and $E E(0,0)$ are locus $I I$ and $E E$ without social contract $\left(m_{t}^{*}, \tau_{t+1}^{o *}\right)$ in all periods. The dashed directions of motion also present the dynamics in the case of no social contracts. Similarly, the bold lines $I I\left(m_{t}^{*}, \tau_{t+1}^{o *}\right)$, $E E\left(m_{t}^{*}, \tau_{t+1}^{o *}\right)$ and the bold directions of motion represent corresponding locus and dynamics in the case of having social contracts $\left(m_{t}^{*}, \tau_{t+1}^{o *}\right)$.

In the case $\hat{I}<\tilde{I}$, under the period-by-period intergenerational social contracts $\left(m_{t}^{*}, \tau_{t+1}^{o *}\right)_{t}$ the economy has a chance to converge to steady state characterized by $(I, E)$ in which $E<\tilde{E}$ as depicted in Figure 3. Interestingly, in this case, under period-by-period social contracts $\left(m_{t}^{*}, \tau_{t+1}^{o *}\right)_{t}$ determined through Nash bargaining, the steady state $(\tilde{I}, \tilde{E})$ disappears and the dynamics of motion change substantially.

In the case $\hat{I}>\tilde{I}$ and the cardinal $\left\|E E\left(m_{t}^{*}, \tau_{t+1}^{o *}\right) \cap I I\left(m_{t}^{*}, \tau_{t+1}^{o *}\right)\right\| \geq 1$, then under the period-by-period intergenerational social contracts $\left(m_{t}^{*}, \tau_{t+1}^{o *}\right)_{t}$ the economy has a chance to converge to a better steady state characterized by $(I, E)$ in which not only $E<\tilde{E}$ but also $I>\tilde{I}$ as depicted in Figure 4. Interestingly, in this case, unlike when $\hat{I}<\tilde{I}$, under the period-by-period social contracts $\left(m_{t}^{*}, \tau_{t+1}^{o *}\right)$ the steady state without social contracts does not vanish. Because the steady state $(\tilde{I}, \tilde{E})$ is stable, as proved in proposition 3 , in the case $\hat{I}>\tilde{I}$, there always exists an area such that when the economy enters this area, it will 


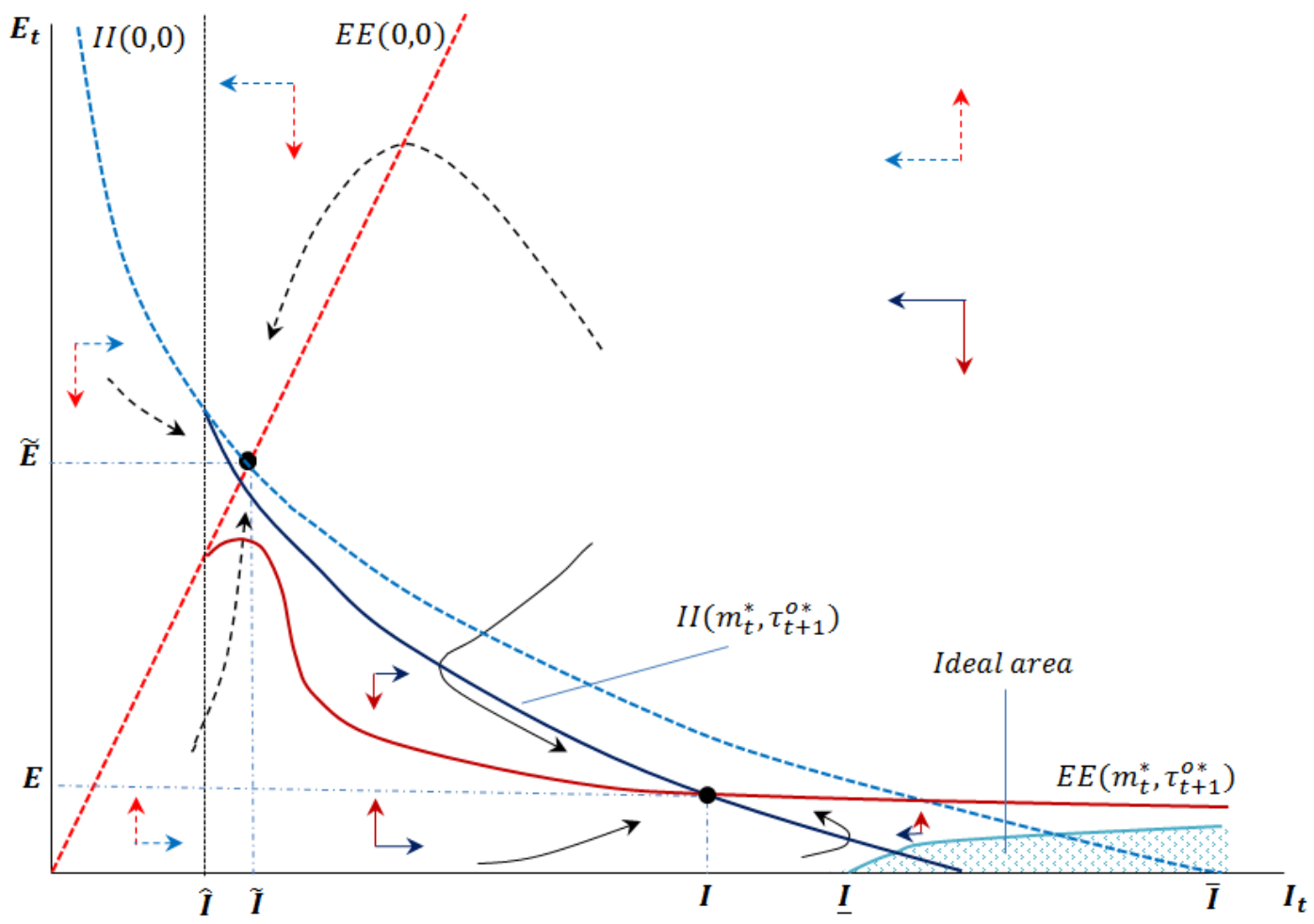

Figure 3: Dynamics and steady states in the case $\hat{I}<\tilde{I}$

converge to the stable steady state $(\tilde{I}, \tilde{E})$ and the social contracts in this setup cannot help the economy escape this trap. For this reason, we call this area the "dangerous area". Figure 4 depicts the dynamic behavior of the economy and it also characterizes the dangerous area (shaded) if the convergence behavior to the steady state without social contract, $(\tilde{I}, \tilde{E})$, is not spiral. This result reveals an important economic implication: If the economy delays signing intergenerational social contracts when its income is still sufficiently high, the costs of this delay may be extremely high, particularly when the economy enters the dangerous area. 


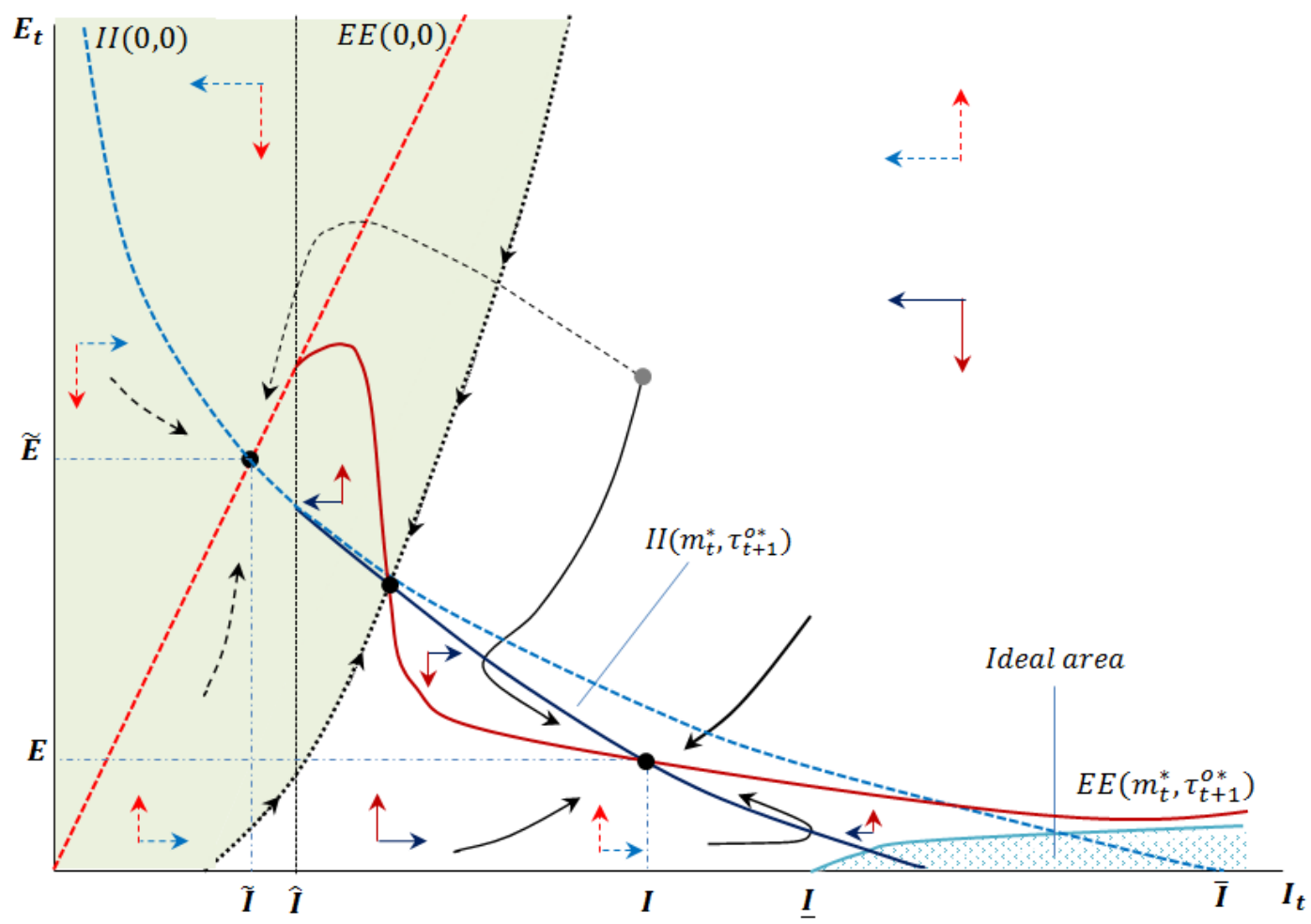

Figure 4: Dynamics and steady states in the case $\hat{I}>\tilde{I}$

\section{Simulation}

In the previous section, we argue that through Pareto-improving intergenerational social contracts, an economy may have a chance to converge to a better steady state with lower stationary pollution stock and higher stationary income compared to the case of no social contracts. In this section we provide numerical simulation results to illustrate the argument in the previous section. The share of capital, $\alpha$, is set to 0.3 which is quite well known from the literature. The rate of time preference, $\beta$, is set to 0.7 which gives us a plausible savings/income rate of households around $40 \%$. Without loss of generality we set the effectiveness of mitigation to $\gamma=1$. Note that these paraeter values guarantee that the condition in assumption 1 holds. With the above parameters values we compute $\hat{I}=\frac{\alpha(1+\alpha \beta)(1+\beta)^{2}}{(1-\alpha)(\beta+\gamma+\gamma \beta) \beta^{2}} \simeq 1.2744$. We run the simulation with different levels of technology $A$ corresponding to the two distinct cases $\tilde{I}<\hat{I}$ and $\tilde{I}>\hat{I}$ in the following subsections. 


\subsection{The case $\hat{I}<\tilde{I}$}

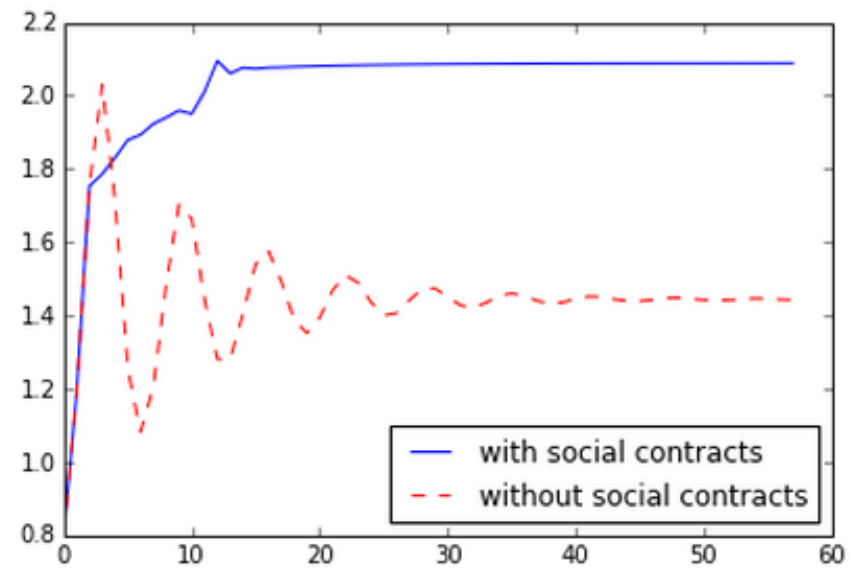

Figure. Income dynamics with and without social contracts in the case $\hat{I}<\tilde{I}$

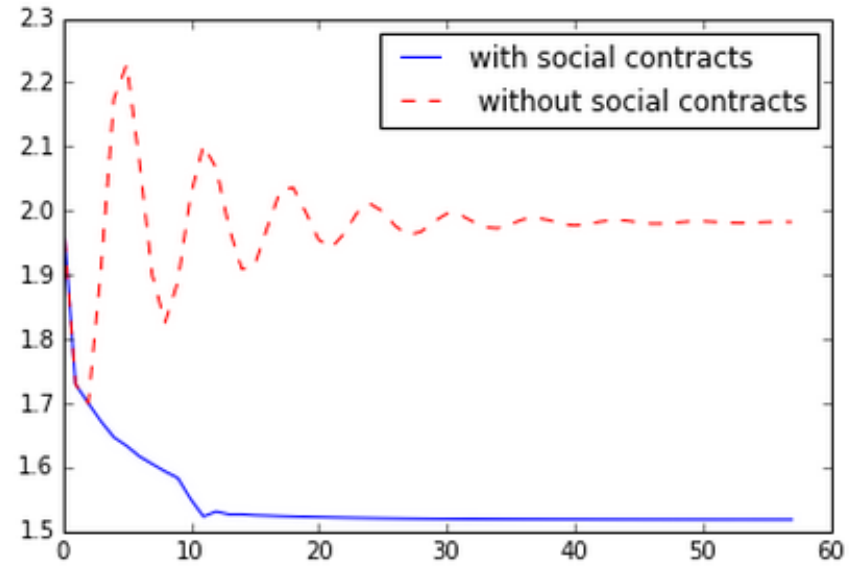

Figure. Pollution stock dynamics with and without social contracts in the case $\hat{I}<\tilde{I}$

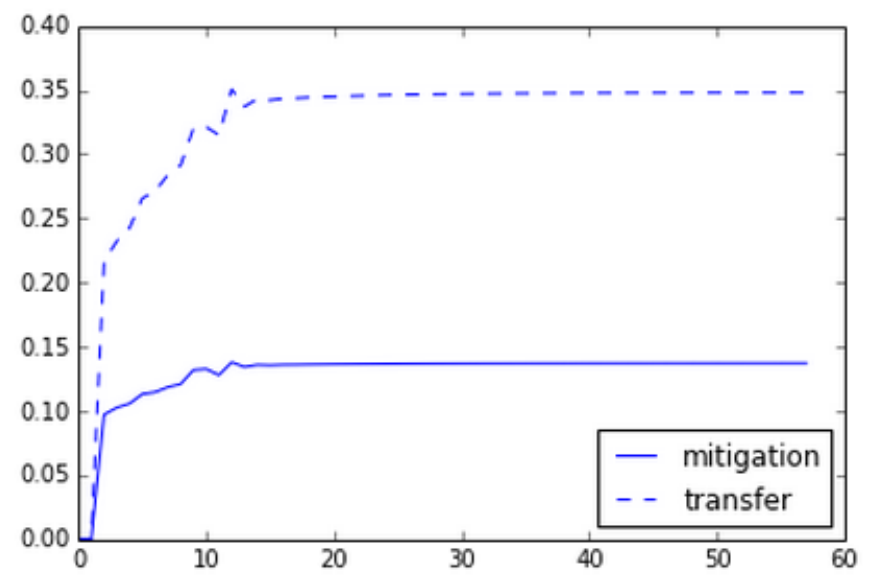

Figure. Mitigation and transfer in the case $\hat{I}<\tilde{I}$ 


\subsection{The case $\hat{I}>\tilde{I}$}

\section{Early signing of social contracts}

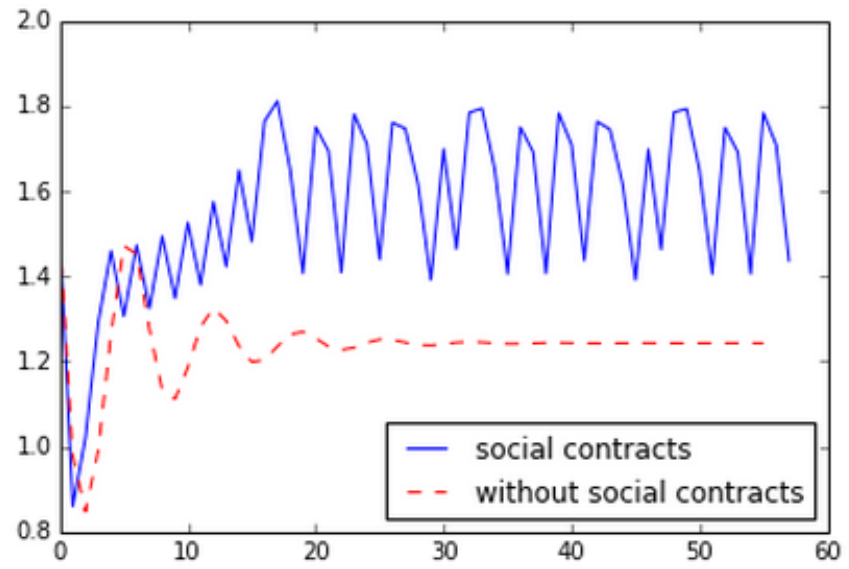

Figure. Income dynamics with and without social contracts in the case $\hat{I}>\tilde{I}$

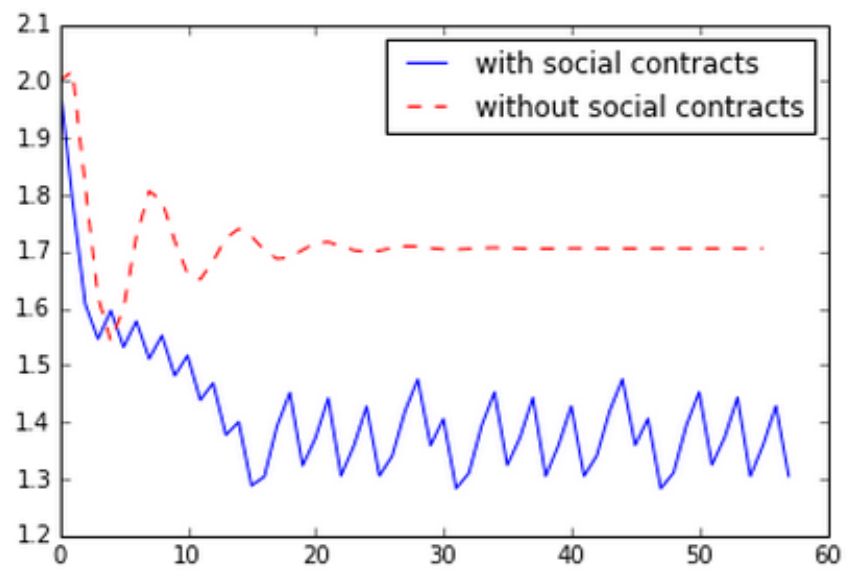

Figure. Pollution stock dynamics with and without social contracts in the case $\hat{I}>\tilde{I}$

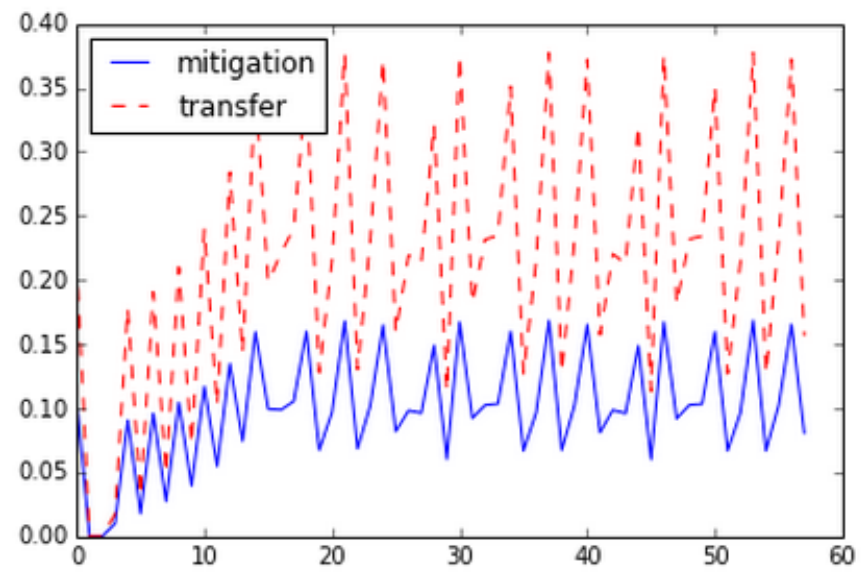

Figure. Mitigation and transfer in the case $\hat{I}>\tilde{I}$ 


\section{Delayed signing of social contracts}

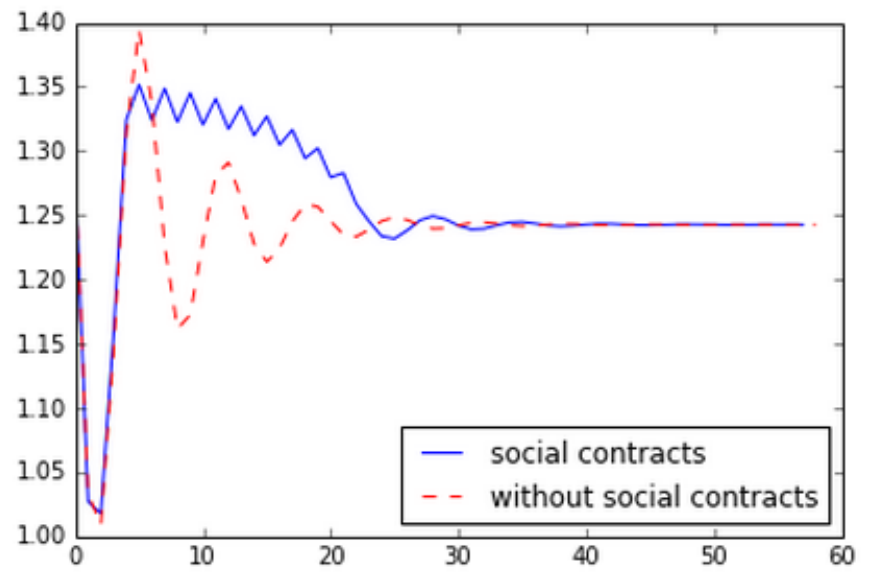

Figure. Income dynamics when delaying to sign social contracts in the case $\hat{I}>\tilde{I}$

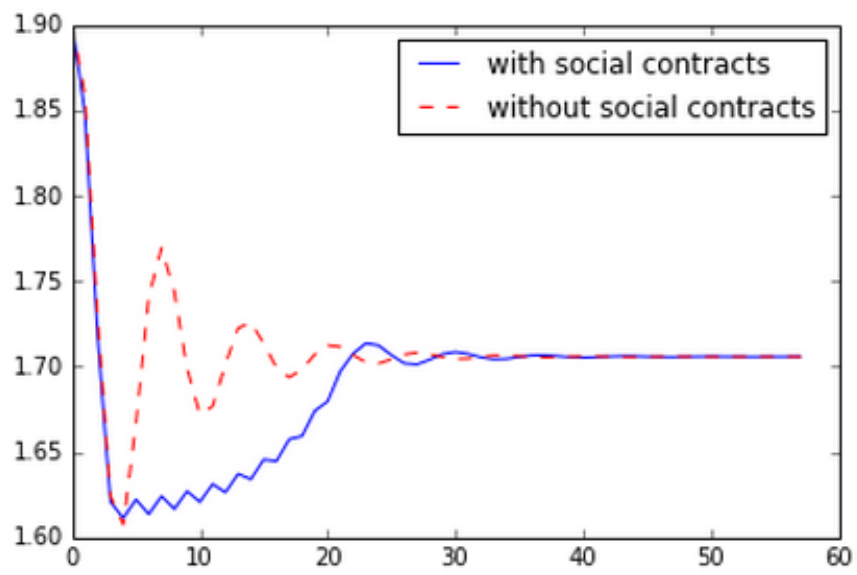

Figure. Pollution stock dynamics when delaying to sign social contracts in the case $\hat{I}>\tilde{I}$

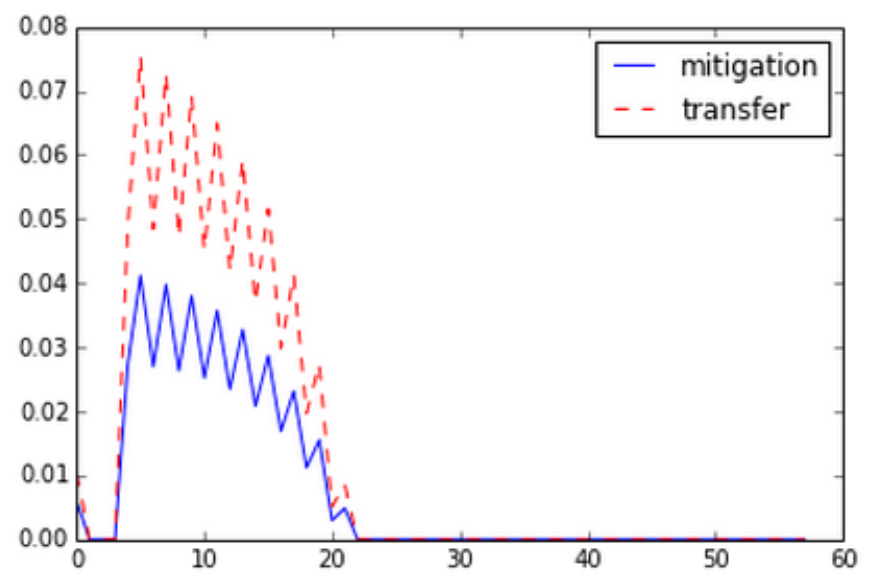

Figure. Mitigation and transfer in the case $\hat{I}>\tilde{I}$ 
In the previous section, when $\hat{I}>\tilde{I}$ we ignored the case $E E\left(m_{t}^{*}, \tau_{t+1}^{o *}\right) \cap I I\left(m_{t}^{*}, \tau_{t+1}^{o *}\right)=\oslash$. In this case, even under period-by-period social contracts, at the end the economy converges to the globally stable steady state $(\tilde{I}, \tilde{E})$. This case implies that the social contracts are not strong enough to help the economy avoid the steady state $(\tilde{I}, \tilde{E})$. In this paper, we do not focus on this case in both analytical analysis and simulation because, in our point of view, this case can be ruled out when we extend the model by allowing for technological progress. Technical progress can increase conditional steady state income $\tilde{I}$ above the threshold $\hat{I}$.

\section{A non-cooperative setup}

So far, it has been assumed that two generations $t$ and $t+1$ determine a contract $\left(m_{t}^{*}, \tau_{t+1}^{o *}\right)$ through negotiation at the beginning of period $t+1$. An agent in his working-age in period $t$ leaves part of his labor income from period $t$ to the next generation to cover mitigation expenses. In exchange, he expects a subsidy on his old-age capital income. This setup neglects any commitment problems of agents, reflecting the idea that the legal framework and institutions allow to enforce compliance. Legal enforcement may however be problematic for intergenerational contracts as we consider them here, as they usually involve generations not legally of age or not even born. If agents of the young generation have an incentive to default on their obligations from the contract once the mitigation level is set, a contract between generations - even if Pareto-improving for both - will not be sustainable over time without effective enforcement. In this section, we explicitly account for commitment problems and characterize 'self-enforcing' contracts which are sustainable over time even in the absence of external enforcement mechanisms.

Consider the contract between the adult generations in $t$ and $t+1$ : If both generations know that there will not be another contract after period $t+1$, the working-age generation in $t+1$ will always want to default on its contract with generation $t$ if effective enforcement is not in place. From the perspective of generation $t+1$, the mitigation level $m_{t}$ is given and independent of whether or not it pays the transfer to generation $t$. Having perfect foresight, generation $t$ will anticipate that it will not receive a transfer. It will therefore not offer a contract in the first place and choose not to mitigate. For there to be a chance that a contract scheme will be implemented and sustained despite commitment problems, there has to be a return from complying with the contracts. This can only be the case if each generation $t+1$ expects the next generation to sign a similar contract granting them a transfer as well but only if they comply. It has been shown e.g. by Rangel (2003), Cigno (1993, 2006a, 2006b) and Anderberg and Balestrino (2003) that if the return to participating in such a system of intergenerational transfers, i.e. the difference between the transfer paid and the transfer received, is sufficiently large, it can ensure financing of a public good like education or environmental quality. With lump sum transfers, however, Cigno as well as Anderberg and Balestrino show that population 
growth is needed to make the transfer system attractive compared to other forms of investment and that in this case, private investment is crowded out. We show that in our setup, contracts can be incentive compatible in the absence of population growth and that investment in the transfer scheme and in physical capital are to some extent complementary.

In the following, we first transform the problem of finding period-by-period contracts between successive overlapping generations into a strategic game. We set up conditions under which the working-age generation in a period $t$ will not default on a social contract forcing it to invest an amount $\widehat{m}_{t}$ in mitigation and transfer a share $\hat{\tau}_{t}^{y}$ of its income to provide for the subsidy $\widehat{\tau}_{t}^{o}$ to the capital return of the old in $t$. These conditions naturally depend on the expected transfer $\tau_{t+1}^{o, e}$ and on whether previous generations chose to comply with the contract. We then analyze the existence of equilibrium paths with positive transfer payment and mitigation investment in each period satisfying these conditions. The associated contract schemes must be subgame perfect equilibria of the dynamic game between generations. In the next step, we examine whether and under which conditions it is possible to find combinations of the mitigation share and transfer, for which a contract scheme is simultaneously Pareto improving and self-enforcing.

\subsection{Incentive constraints and self-enforcing contract schemes}

We now follow Rangel (2003) in formulating the problem of finding incentive compatible intergenerational contracts as a strategic game between two successive generations, which is repeated over time with alternating players. ${ }^{8}$ The following definition clarifies the terminology:

Definition 1. The history $h_{t}, t \geq T$ of a game starting in period $T$ denotes the vector of actions $\left(\left(m_{t-1}, \tau_{t-1}^{y}\right), \ldots,\left(m_{T}, \tau_{T}^{y}\right)\right.$ chosen up to time $t$. A strategy $s_{t}$ for generation $t$ is a plan which assigns a choice $\left(m_{t}, \tau_{t}^{y}\right)$ to every given history $h_{t}=\left(\left(m_{t-1}, \tau_{t-1}^{y}\right), \ldots,\left(m_{T}, \tau_{T}^{y}\right)\right)$. A strategy profile is a set $\left\{s_{t}\right\}_{t=T}^{\infty}$ containing the strategies chosen in each period $t$.

If a mitigation-transfer path imposed by a series of contracts gives no generation an incentive to default from any of the contracts it is involved $\mathrm{in}^{9}$, it is sustainable without external enforcement (by a government). We call such a path self-enforcing. Clearly, a mitigationtransfer path $\left(m_{t}, \tau_{t+1}^{o}\right)_{t=T}^{\infty}$ from some date $T$ onward is self-enforcing if, for each generation and given the history of actions taken by previous generations (in particular given their mitigation investment) and expectations about future generations' actions, complying with the contracts yields higher lifetime-utility than defaulting. Such a path $\left(m_{t}, \tau_{t+1}^{o}\right)_{t=T}^{\infty}$ constitutes a subgame perfect equilibrium of the dynamic game starting at $T$ :

\footnotetext{
${ }^{8}$ We assume here that each generation consists of a large number of identical agents, to be able to neglect free-riding within a generation.

${ }^{9}$ Note that every generation $t$ signs two contracts, one with the previous working-age generation $t-1$ and one with the next working-age generation $t+1$.
} 
Definition 2. An equilibrium path with positive mitigation investment and a positive intergenerational transfer imposed by a series of contracts starting in some period $0<T<\infty$ is self-enforcing if and only if the contract scheme $\left(m_{t}, \tau_{t+1}^{o}\right)_{t=T}^{\infty}$ is a subgame perfect equilibrium of the infinitely repeated game between overlapping generations.

While the old generation in a period $t$ has no obligations but only benefits from a contract, the young generation faces a trade-off. To make the existence of self-enforcing schemes possible, two ingredients are crucial: (1.) There must not be a known terminal date: As explained above, a single contract between two generations $t$ and $t+1$ will not be sustainable if both generations know future generations at some point in time will not sign another contract. ${ }^{10}$ (2.) There has to be credible punishment for deviation from a contract ${ }^{11}$ : Generation $t$ 's reward for compliance is the transfer it receives from generation $t+1$ when old. But it will only have an incentive to comply if it does not receive this transfer also if it deviates. Generation $t+1$ must thus have an incentive to punish generation $t$ for defaulting.

As Rangel (2003) proves in his paper, a path of mitigation investment and transfers can be sustained as a subgame perfect equilibrium in an infinitely repeated game if and only if it can be sustained by what he calls 'simple trigger strategies'. ${ }^{12}$ These are strategies under which each deviation is punished in the next - but only in the next - period. The following simple trigger strategy $s_{t}\left(h_{t}\right)$ is a slight modification of the one in Rangel (2003), where $\left(\hat{m}_{t}, \hat{\tau}_{t}^{y}\right)$, $\hat{m}_{t}, \hat{\tau}_{t}^{y}>0$ denotes some given combination of mitigation investment and transfer payment to the current old:

$$
s_{t}\left(h_{t}\right)= \begin{cases}\left(\hat{m}_{t}, \hat{\tau}_{t}^{y}\right) & \text { if } p\left(h_{t}\right)=C \\ \left(\hat{m}_{t}, 0\right) & \text { if } p\left(h_{t}\right)=P\end{cases}
$$

where

$$
p\left(h_{t}\right)= \begin{cases}C & \text { if }\left(m_{t-1}, \tau_{t-1}^{y}\right)=\left(\hat{m}_{t-1}, \hat{\tau}_{t-1}^{y}\right) \text { and } p\left(h_{t-1}\right)=C \\ C & \text { if }\left(m_{t-1}, \tau_{t-1}^{y}\right)=\left(\hat{m}_{t-1}, 0\right) \text { and } p\left(h_{t-1}\right)=P \\ P \quad \text { if } t=T \\ P \quad \text { otherwise }\end{cases}
$$

$p\left(h_{t}\right)=C$ indicates that the game is in a compliance phase. This phase occurs (i) if generation $t-1$ has invested in mitigation and paid the transfer to generation $t-2$ and generation $t-2$ has itself complied with the contract and (ii) if generation $t-1$ has invested in mitigation but not paid the transfer because generation $t-2$ has deviated. $p\left(h_{t}\right)=P$ occurs if generation $t-1$ has deviated, so that the game is in a punishment phase.

As proven in Rangel (2003), it is sufficient to prove that the strategy defined above is

\footnotetext{
${ }^{10}$ As Boldrin and Rustichini (2000) point out, if the time is not known with certainty, generations may choose compliance even if they are aware that the mitigation-transfer scheme will not be sustained forever.

${ }^{11}$ This point has, amongst others, been pointed out by Rangel (2003).

${ }^{12}$ We refer the interested reader to proposition 1 in Rangel (2003).
} 
a subgame perfect equilibrium of the game, which we will do in the next paragraph. If a generation $t$ wants to deviate, the best possible deviation is to choose $\left(m_{t}, \tau_{t}^{y}\right)=(0,0)$. This is because if it chooses either the transfer to the currently old or mitigation different from the level prescribed by the contract, it will be punished by the next adult generation. It should thus expect not to receive a transfer when old $\left(\tau_{t+1}^{o, e}=0\right)$ and therefore choose both mitigation and the transfer payment equal to zero. If generation $t$ complies, on the contrary, it can expect to obtain the transfer it settles in the contract with generation $t+1$, i.e. $\tau_{t+1}^{o, e}=\hat{\tau}_{t+1}^{o}$.

Denote expected indirect utility from compliance as $V^{c}\left(\hat{m}_{t}, \tau_{t}^{y} i p\left(h_{t}\right), \tau_{t+1}^{o, e}=\hat{\tau}_{t+1}^{o}\right)$, where $\tau_{t}^{y} \epsilon\left[0, \hat{\tau}_{t}^{y}\right]$ depending on the state $p\left(h_{t}\right)$ of the game. Expected indirect utility from deviation is $V^{d}\left(0,0: p\left(h_{t}\right), \tau_{t+1}^{o, e}=0\right)$. Generation $t$ compares indirect utilities for any given history $h_{t}$, that is for the case that the previous generation complied and for the case that it deviated.

\section{Incentive constraint when the state of the game is compliance}

When the game is in a compliance phase $\left(p\left(h_{t}\right)=C\right)$ and generation $t$ complies with the contract, indirect utility is:

$$
\begin{array}{r}
V^{c}\left(\hat{m}_{t}, \hat{\tau}_{t}^{y} \mid p\left(h_{t}\right)=C, \tau_{t+1}^{o, e}=\hat{\tau}_{t+1}^{o}\right)=\Phi+\beta \ln \left[z\left(E_{t}\right) \alpha\left(\frac{\beta I_{t}^{C}\left(1-\hat{m}_{t}\right)}{1+\beta}\right)^{\alpha-1}\right] \\
+(1+\beta)\left[\ln I_{t}^{C}+\ln \left(1-\hat{m}_{t}\right)\right]+\beta \ln \left(1+\hat{\tau}_{t+1}^{o}\right)
\end{array}
$$

where $\Phi=\ln \frac{1}{1+\beta}+\beta \ln \frac{\beta}{1+\beta}, I_{t}^{C}=z\left(E_{t-1}\right)(1-\alpha) k_{t}^{\alpha}\left(1-\hat{\tau}_{t}^{y}\right)$ is the income of the young agent in period $t$ after paying $z\left(E_{t-1}\right)(1-\alpha) k_{t}^{\alpha} \hat{\tau}_{t}^{y}=\hat{\tau}_{t}^{o} R_{t} k_{t}$ to the old in $t$ and $\hat{\tau}_{t+1}^{o}$ is the foreseen transfer that generation $t$ will receive from generation $t+1$. Recall that the pollution stocks $E_{t-1}$ and $E_{t}$ as well as the capital stock $k_{t}$ are given in period $t .{ }^{13}$

If generation $t$ deviates, indirect utility is

$$
V^{d}\left(0,0 \text { ip }\left(h_{t}\right)=C, \tau_{t+1}^{o, e}=0\right)=\Phi+\beta \ln \left[z\left(E_{t}\right) \alpha\left(\frac{\beta I_{t}^{D}}{1+\beta}\right)^{\alpha-1}\right]+(1+\beta) \ln I_{t}
$$

where $I_{t}^{D}=z\left(E_{t-1}\right)(1-\alpha) k_{t}^{\alpha}$ is income if generation $t$ does not pay the transfer $\left(\hat{\tau}_{t}^{y}=0\right)$.

The difference between indirect utilities is

$$
\triangle V\left(\hat{m}_{t}, \hat{\tau}_{t}^{y} \mid p\left(h_{t}\right)=C, \hat{\tau}_{t+1}^{o}\right)=(1+\alpha \beta) \ln \left(\left(1-\hat{m}_{t}\right)\left(1-\hat{\tau}_{t}^{y}\right)\right)+\beta \ln \left(1+\hat{\tau}_{t+1}^{o}\right) .
$$

There is no incentive to deviate if and only if

\footnotetext{
${ }^{13}$ The stock variables $E$ and $k$ and therefore also income $I$ and the interest rate of course depend on the complete history of the game. We will see below however that they do not affect generation $t^{\prime} s$ decision to comply. For means of simplification, we therefore neglect the history dependence of $E, k$ and $I$ in the following.
} 


$$
\triangle V\left(\hat{m}_{t}, \hat{\tau}_{t}^{y_{i}} p\left(h_{t}\right)=C, \hat{\tau}_{t+1}^{o}\right) \geq 0
$$

or, equivalently,

$$
\left[\left(1-\hat{m}_{t}\right)\left(1-\hat{\tau}_{t}^{y}\right)\right]^{1+\alpha \beta}\left(1+\hat{\tau}_{t+1}^{o}\right)^{\beta} \geq 1 .
$$

Using the relation $z\left(E_{t-1}\right)(1-\alpha) k_{t}^{\alpha} \hat{\tau}_{t}^{y}=\hat{\tau}_{t}^{o} R_{t} k_{t}$ between the transfer received by the old generation in $t$ and the transfer paid by the working-age generation in $t$, this condition can be restated as

$$
\left(1-\frac{\alpha}{1-\alpha} \hat{\tau}_{t}^{o}\right)^{1+\alpha \beta}\left(1-\hat{m}_{t}\right)^{1+\alpha \beta}\left(1+\hat{\tau}_{t+1}^{o}\right)^{\beta} \geq 1 .
$$

Note that the constraint does not depend on the size of the transfer or mitigation shares before period $t$, nor on any stock variable.

\section{Incentive constraint when the state of the game is punishment}

Now consider the decision of generation $t$ if generation $t-1$ deviated from the contract, choosing $\left(m_{t-1}, \tau_{t-1}^{y}\right)=(0,0)$. In this case, generation $t$ should punish generation $t-1$ with the choice $\left(\hat{m}_{t}, 0\right)$.

Indirect utility from compliance with the contract when the game is in a punishment phase $\left(p\left(h_{t}\right)=P\right)$ is:

$$
\begin{aligned}
& V^{c}\left(\hat{m}_{t}, 0^{\prime} p\left(h_{t}\right)=P, \tau_{t+1}^{o, e}=\hat{\tau}_{t+1}^{o}\right)=\Phi+\beta \ln \left[z\left(E_{t}\right) \alpha\left(\frac{\beta I_{t}^{C}\left(1-\hat{m}_{t}\right)}{1+\beta}\right)^{\alpha-1}\right] \\
& +(1+\beta)\left[\ln I_{t}^{C}+\ln \left(1-\hat{m}_{t}\right)\right]+\beta \ln \left(1+\hat{\tau}_{t+1}^{o}\right)
\end{aligned}
$$

where $I_{t}^{C}=z\left(E_{t-1}\right)(1-\alpha) k_{t}^{\alpha}$ is income if the agent born in $t$ chooses compliance.

Indirect utility from deviation is:

$$
V^{d}\left(0,0 \operatorname{li}\left(h_{t}\right)=P, \tau_{t+1}^{o, e}=0\right)=\Phi+\beta \ln \left[z\left(E_{t}\right) \alpha\left(\frac{\beta I_{t}^{D}}{1+\beta}\right)^{\alpha-1}\right]+(1+\beta) \ln I_{t}
$$

where $I_{t}^{D}=z\left(E_{t-1}\right)(1-\alpha) k_{t}^{\alpha}=I_{t}^{C}$. Note that income is the same whether generation $t$ complies with the contract or deviates because in a punishment phase, generation $t$ will not pay the transfer even if it chooses compliance.

There is no incentive for deviation if and only if:

$$
\triangle V\left(\hat{m}_{t}, 0_{p}^{\prime} p\left(h_{t}\right)=P_{t}, \hat{\tau}_{t+1}^{o}\right)=(1+\alpha \beta) \ln \left(1-\hat{m}_{t}\right)+\beta \ln \left(1+\hat{\tau}_{t+1}^{o}\right) \geq 0
$$


This condition is equivalent to

$$
\left(1-\hat{m}_{t}\right)^{1+\alpha \beta}\left(1+\hat{\tau}_{t+1}^{o}\right)^{\beta} \geq 1
$$

Not surprisingly, the minimum expected transfer $\hat{\tau}_{t+1}^{o}$ required by (37) to make the adult agent comply with the contract for a given $\hat{m}_{t}$ is smaller than that of (36) whenever $\hat{\tau}_{t}^{o}>0$ : To receive a transfer in period $t+1$ when the history of the game is compliance, the adult agent has to pay a share $\hat{\tau}_{t}^{y}=\frac{\alpha}{1-\alpha} \hat{\tau}_{t}^{o}$ of his income in addition to mitigation costs, while he does not have to pay the transfer in the punishment phase. Therefore, the expected transfer $\hat{\tau}_{t+1}^{o}$ which is needed to make the agent just indifferent between compliance and default is higher after a history of compliance.

The insights of this paragraph lead to the following lemma:

Lemma 5. A path with positive mitigation investment $\hat{m}_{t}>0$ compensated for by a positive transfer $\hat{\tau}_{t+1}^{o}>0$ in every period $t=T \ldots \infty$ can be sustained as a subgame perfect equilibrium for the game with initial state $p\left(h_{T}\right)=P$ if and only if condition (37) is satisfied in period $t=T$ and (36) is satisfied in every period $t>T$, with $\tau_{t+1}^{o, e}=\hat{\tau}_{t+1}^{o}$.

Proof. The proof is contained in the text.

\subsection{Existence of self-enforcing mitigation-transfer schemes}

We have defined the conditions for an equilibrium path with mitigation and an intergenerational transfer to be self-enforcing. Now the question arises if there exist schemes $\left(m_{t}, \tau_{t+1}^{o}\right)_{t=T}^{\infty}$ with $m_{t}>0, \tau_{t+1}^{o}>0$ which satisfy the conditions in every period $t \geq T$.

The first conclusion in this regard must be that along an equilibrium path with self-enforcing contracts, the intergenerational transfer and the mitigation share must converge to or stay in the vicinity of some constant values $\tau^{o}, m$. Neither can they decrease persistently while still satisfying $m_{t}>0, \tau_{t+1}^{o}>0$. Nor can they increase unboundedly: At the latest if all income has to be given up to pay the transfer or invest in mitigation, the contract scheme will break down. The existence of a non-empty set of stationary pairs $\left(m, \tau^{o}\right)$ with $m>0, \tau^{o}>0$ which satisfy the incentive constraints is therefore both necessary and sufficient for the existence of self-enforcing mitigation-transfer schemes.

We can characterize the set of self-enforcing mitigation-transfer schemes with stationary transfer $\tau_{t+1}^{o}=\tau_{t}^{o}=\tau^{o}$ for all $t$ by setting $\tau_{t+1}^{o}=\tau_{t}^{o}=\tau^{o}$ in condition (36) and rearranging:

$$
S^{I C}=\left\{\left(m, \tau^{o}\right) \in[0,1) \times\left[0, \tau^{o, \max }\right): m \leq 1-\frac{1}{\left(1-\frac{\alpha}{1-\alpha} \tau^{o}\right)\left(1+\tau^{o}\right)^{\frac{\beta}{1+\alpha \beta}}}\right\}
$$

We defined as $\tau^{o, \max } \equiv \frac{1-\alpha}{\alpha}$ the maximum transfer reconcilable with non-negative income (i.e. 
$\left.\tau_{t}^{y} \leq 1\right)$ of the working-age agent in period $t$. In what follows, we call this set the 'stationary' incentive compatible set and its boundary, given by

$$
m^{I C}=1-\frac{1}{\left(1-\frac{\alpha}{1-\alpha} \tau^{o}\right)\left(1+\tau^{o}\right)^{\frac{\beta}{1+\alpha \beta}}} \forall t
$$

the 'stationary' incentive compatibility constraint.

Lemma 6. Along an equilibrium path with self-enforcing contracts from a period $T$ onwards, the series $\left(m_{t}, \tau_{t+1}^{o}\right)_{t=T}^{\infty}$ must converge to or fluctuate without trend around a constant $\left(m, \tau^{o}\right)$. The set of stationary self-enforcing pairs $\left(m, \tau^{\circ}\right)$ is characterized by (38).

Proof. The proof is contained in the text.

A necessary and sufficient condition for the existence of a non-empty stationary set $S^{I C}$ and thus the existence of self-enforcing contracts is the following: In a situation without a social contract ( so that $m_{t}=\tau_{t}^{o}=\tau_{t+1}^{o}=0$ ) the marginal utility gain from an increase in the transfer received must exceed the marginal utility loss from an equal increase in the transfer paid. ${ }^{14}$ Graphically, the slope of the stationary incentive constraint at $m=\tau^{o}=0$ must be positive. Formally, this is guaranteed by the following condition:

$$
\beta>\frac{\alpha}{1-\alpha}(1+\alpha \beta)
$$

Proposition 5. There exist pairs $\left(m_{t}, \tau_{t+1}^{o, e}\right)$ with $\tau_{t+1}^{o, e}=\tau_{t+1}^{o}$ and $m_{t}>0, \tau_{t+1}^{o}>0$ for every $t=T \ldots \infty$ such that the associated equilibrium path of contracts from period $T$ onwards is self-sustainable as a subgame perfect equilibrium if and only if condition (40) is satisfied. The maximum mitigation share which can be sustained is strictly smaller than one.

Proof. See Appendix A7.

The left hand side of condition (40) gives the marginal gain $\beta$ in indirect utility of marginally raising the transfer received, $\tau^{o}$, from zero. On the right hand side, $1+\alpha \beta$ is the net marginal decrease in utility due to an increase in the transfer paid, $\tau^{y}$, by one marginal unit: The transfer paid reduces current income. It thereby lowers the level of savings and the future capital stock as well as the total return to savings for a given interest rate. This results in a marginal decrease in indirect utility by $1+\beta$. On the other hand, reduced capital accumulation increases the equilibrium interest rate $R_{t+1}$. This raises indirect utility by $(1-\alpha) \beta$. The sum of the two effects is $1+\alpha \beta$. Finally, the ratio $\frac{\alpha}{1-\alpha}$ states by how much the transfer paid, $\tau^{y}$, has to rise to finance a one unit increase in the transfer rate $\tau^{o}$ to the current old.

\footnotetext{
${ }^{14}$ See also Rangel (2003) for a similar result.
} 
Note that for the existence of a sustainable path, it is crucial that the ratio $\frac{\alpha}{1-\alpha}$, which is the ratio of capital- to labor income at $m_{t}=\tau_{t}^{o}=\tau_{t+1}^{o}=0$, must be strictly smaller than one. For a transfer scheme to generate a net marginal utility gain, in particular given discounting of future consumption flows and opportunity costs in terms of foregone capital investment, it has to yield a sufficiently high return. Contrary to the previously cited models by Cigno (1993, 2006a, 2006b) and Anderberg and Balestrino (2003), we do not assume positive population growth. However, population growth is typically the only mechanism guaranteeing a positive rate of return to intergenerational transfers if they are granted lump sum. In our model, the transfer is substracted from labor income of the working-age generation and given to the current old as a subsidy on capital returns. Therefore, the transfer rate $\tau^{y}$ the working-age generation has to pay to provide (and later receive) a given transfer rate $\tau^{o}$ on old-age income is the smaller, the smaller the old generation's capital income compared to the working-age generation's labor income. In particular, as long as $\frac{\alpha}{1-\alpha}<1$, the transfer rate paid is lower than the transfer rate received, i. e. $\tau^{y}=\frac{\alpha}{1-\alpha} \tau^{o}<\tau^{o}$. This gives rise to a positive net return of investing in a system of intergenerational transfers even in the absence of population growth. For the usual values $\alpha=1 / 3,1-\alpha=2 / 3$ of the capital and labor elasticities in the production function, $\frac{\alpha}{1-\alpha}$ is indeed below one so that condition (40) can be satisfied.

Instead of motivating condition (40) looking at marginal utility effects of a contract, we can also derive it by comparing real returns from pure capital investment and capital investment combined with the transfer scheme ${ }^{15}$ : The scheme of intergenerational transfers we propose here is comparable to an investment from the viewpoint of an agent of a working-age generation. As in the previously cited literature with lump sum intergenerational transfers, investment in the transfer system competes with the accumulation of physical capital for given income. Our assumption that transfers are granted as subsidy to the return to savings in physical capital ensures however that there cannot be a return to the intergenerational transfer without capital investment. Further, the return to an investment in the transfer system is the higher, the higher capital investment and vice versa. This complementarity is absent in models with lump sum transfers and avoids the complete crowding out of private investment by the transfer scheme. Condition (40) then demands that in a situation without a social contract (so that $m_{t}=\tau_{t}^{o}=\tau_{t+1}^{o}=0$ ) the net return of marginally increasing $\tau_{t}^{o}$ to invest in a transfer scheme in addition to investing in capital is strictly larger than the return that could be obtained by investing solely in capital instead.

\footnotetext{
15 The comparison can be found in appendix A8.
} 


\subsection{Self-enforcing mitigation and Pareto improvement}

We have pointed out in section 3 that for sufficiently high income, there exist combinations of mitigation investment and transfers which, when implemented, lead to a Pareto improvement between successive generations. We have also shown, in the previous subsection, that there exist equilibrium paths with positive mitigation investment and positive transfers in each period which are self-enforcing if commitment problems are taken into account. We are now interested in the question if and under which condition(s) there exist series of Pareto-improving contracts $^{16}$ between successive generations which are also self-enforcing.

Along an equilibrium path with self-enforcing Pareto improving contracts first implemented in a period $T>0$, conditions (19) and (23) for Pareto improvement as well as condition (36) for incentive compatibility have to be satisfied in every period $t \geq T$. Reinspection of the conditions shows that condition (19), which gives the lower boundary of the Pareto improvement set, is equivalent to condition (37). Both are less strict than condition (36) and can therefore be ignored. We only have to check whether conditions (23) and (36), i. e. conditions

$$
\begin{aligned}
\left(1-\frac{\alpha}{1-\alpha} \tau_{t+1}^{o}\right)^{1+\alpha \beta}\left(1-m_{t}\right)^{\alpha(1+\alpha \beta)} e^{\frac{\beta+\gamma(1+\beta)}{1+\beta} \beta m_{t} I_{t}} & \geq 1 \\
\left(1-m_{t}\right)^{1+\alpha \beta}\left(1+\tau_{t+1}^{o}\right)^{\beta}\left(1-\frac{\alpha}{1-\alpha} \tau_{t}^{o}\right)^{1+\alpha \beta} & \geq 1
\end{aligned}
$$

can be simultaneously satisfied in every period $t \geq T .{ }^{17}$

It is important to note that (23) and (36) have to hold for all $t \geq T$. Understanding when this is the case is not an easy task, due to the dynamic nature of the two conditions: We know from the last section that every self-enforcing contract scheme has to be in the vicinity of some stationary pair $\left(m, \tau^{o}\right)$ in the long run. But even if we concentrate on stationary contracts with constant $m, \tau^{o}$ for all $t$, condition (23) still changes over time with income $I_{t}$. Conditional on $S^{I C} \neq \oslash$, a sufficient condition for the existence of a Pareto improving and self-enforcing mitigation path starting in a period $T>0$ is that for all $t \geq T$, the set $S^{I C}$ intersects the Pareto improvement set $\mathcal{P}_{t+1}^{t}$ and this is correctly anticipated. ${ }^{18}$ Formally, we require $S^{I C} \cap \mathcal{P}_{t+1}^{t} \neq \oslash, \forall t \geq T$.

Figure 5 shows the set $\mathcal{P}_{t+1}^{t}$ of Pareto improving combinations $\left(m_{t}, \tau_{t+1}^{o}\right)$ in period $t$ together with the stationary incentive compatible set $S^{I C}$, in $\left(m_{t}, \tau_{t+1}^{o}\right)$-space:

\footnotetext{
${ }^{16}$ Note that we demand every single contract of the series to be a Pareto improvement. As every generation signs two contracts, a weaker requirement for the series of contracts to be Pareto-improving would be to allow welfare gains in one contract to compensate welfare losses in the other.

${ }^{17} \mathrm{In}$ fact, in period $t=T$, any Pareto-improving contract between generations $T$ and $T+1$ never gives generation $T$ an incentive to deviate. The reason is that $\tau_{T}^{o}=0$ according to our definition of strategies and condition (37) reduces to (19) for $\tau_{t}^{o}=0$.

${ }^{18}$ Because the contract scheme has to be stationary only in the long run, a necessary but not sufficient condition is that $S^{I C} \neq \varnothing$ and the intersection exists and is anticipated to exist for all $t \geq T+s, 0<s<\infty$, that is from some period $T+s$ onwards.
} 

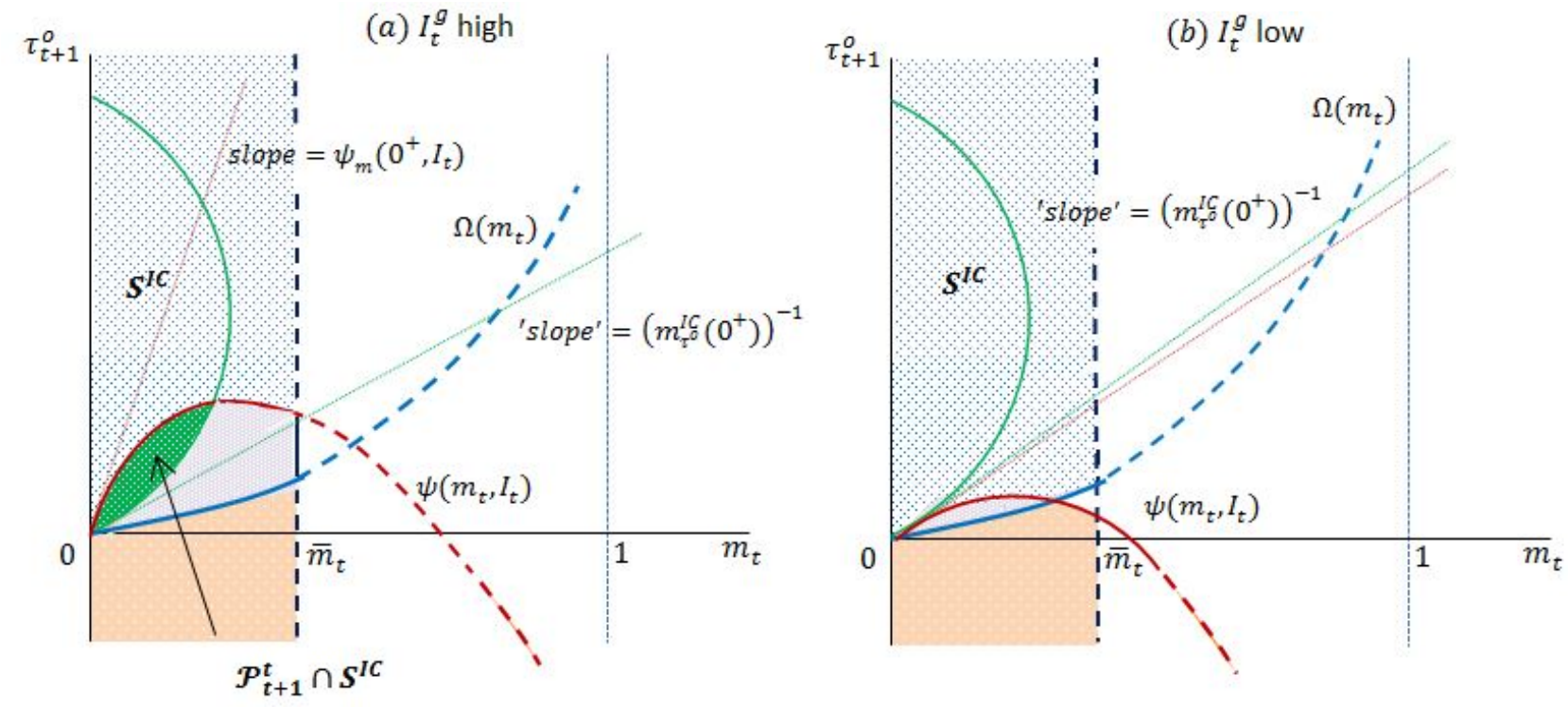

Figure 5: Pareto improvement and incentive compatibility

We will prove below that in a period $t$, conditional on $S^{I C} \neq \oslash, S^{I C} \cap \mathcal{P}_{t+1}^{t} \neq \oslash$ is guaranteed by the following condition on gross income $w_{t} \equiv I_{t}^{g}$ :

$$
I_{t}^{g}>\frac{(1-\alpha) \frac{\beta}{1+\beta}(1+\alpha \beta+\beta)}{\beta-\frac{\alpha}{1-\alpha}(1+\alpha \beta)} \hat{I} \text { where } \beta>\frac{\alpha}{1-\alpha}(1+\alpha \beta)
$$

Recall that $\beta>\frac{\alpha}{1-\alpha}(1+\alpha \beta)$ (condition (40)) guarantees that the set $S^{I C}$ of stationary selfenforcing contracts is non-empty. The new condition (41) on income states that starting from $\left(m_{t}, \tau_{t+1}^{o}\right)=(0,0)$, a slight increase in $m_{t}$ going along with an equal increase $d \tau^{o}$ both in the transfer paid to the current old, $\tau_{t}^{o}$, and the transfer received $\tau_{t+1}^{o}$, such that agents of the transfer-providing generation are just kept from deviating will also improve these agents' welfare compared to the situation without a contract.

Proposition 6. There exist paths $\left(m_{t}, \tau_{t+1}^{o}\right)_{t=T}^{\infty}$ which are both Pareto improving compared to an equilibrium path without a contract and self-enforcing with $\left(m_{t}, \tau_{t+1}^{o}\right)$ being incentive compatible in every $t \geq T$ if along with condition (40), condition (41) holds and is anticipated to hold by the players in every period $t \geq T$.

Proof. See Appendix A9.

Condition (41) is satisfied the easier, the larger the net utility gain of the transfer system, as given by $\beta-\frac{\alpha}{1-\alpha}(1+\alpha \beta)$ and the smaller the income threshold $\hat{I}$ for a Pareto improvement. Due to the effects we described in detail in the previous section and section 3.3, a smaller capital share $\alpha$, a larger weight attached to future consumption as measured by $\beta$, and greater 
efficiency of the mitigation technology $(\gamma)$ have a positive effect on the scope for Paretoimproving, self-enforcing contracts.

\section{Long-run steady state under Pareto improving, self-enforcing contracts}

While the dynamics of income and the pollution stock depend on the particular sequence $\left(m_{t}^{I C, *}, \tau_{t+1}^{o I C, *}\right)_{t=T}^{\infty}$ of contracts chosen, the following result concerning the steady-state holds quite generally:

Proposition 7. Under period-by-period social contracts $\left(m_{t}, \tau_{t+1}^{o}\right), \forall t \geq T$ which are Pareto improving over the situation without a contract and satisfy the incentive constraints of every two generations involved, the steady-state pollution stock will be lower compared to the steadystate without a social contract.

Proof. The isoclines $I I(0,0)$ for income and $E E(0,0)$ for the pollution stock when there are no social contracts are upper boundaries for the respective isoclines following from the system (33) and (34) with social contracts $\left(m_{t}, \tau_{t+1}^{o}\right)$ in all periods. This is true for any pairs $\left(m_{t}, \tau_{t+1}^{o}\right)$ with strictly positive $m_{t}>0, \tau_{t+1}^{o}>0$. Whatever the particular path $\left(m_{t}^{I C, *}, \tau_{t+1}^{o I C, *}\right)_{t=T}^{\infty}$ chosen, this implies that the stock of pollution in a steady-state will be lower under incentive compatible social contracts than under laissez-faire.

So far, we have focussed on conditions for the existence of Pareto-improving, self-enforcing contract paths and their long-run properties. It would be interesting to consider the determination of a particular sequence $\left(m_{t}^{I C, *}, \tau_{t+1}^{o I C, *}\right)_{t=T}^{\infty}$ of contracts via Nash bargaining to study how accounting for incentive compatibility alters the dynamics of income and the pollution stock compared to section 4. It is to be expected that if bargaining leads to a self-enforcing path and the long-run incentive constraint is binding, $m_{t}^{I C, *}$ will not be higher and $\tau_{t+1}^{o I C, *}$ will not be lower compared to the solutions in section 4. Reasoning analogous to the proof above suggests that the steady-state stock of pollution will be higher if incentive constraints are accounted for than when they are neglected.

\section{Conclusion}

Climate change mitigation is a question of intergenerational distribution of costs and benefits and of intergenerational cooperation. While the burden of mitigation mainly falls on current generations, benefits largely accrue to generations not yet born. This is one reason why policy makers biased towards current generations of voters are reluctant to impose environmental policy even if they believe that the correction of the environmental externality is desirable from a social planner's perspective. In this paper, we have studied the scope for mitigation to 
be beneficial for and supported by all generations in a general equilibrium OLG model with non-altruistic agents.

We have considered a social contract between successive generations according to which young generations invest a share of their labor income in mitigation in return for a subsidy to their old-age capital income which is paid by the next young generation. We have derived a condition for the existence of contracts which are Pareto improving over the equilibrium without contract and characterized the set of Pareto improving combinations of mitigation share and transfer, as well as the Nash bargaining solution. While in our model the long-run effects of a social contract on the environment are a priory ambiguous because of its diverse effects on capital accumulation, we have proven that steady-state pollution stock is in fact lower and steady state income is higher under period-by-period social contracts with Nash bargaining.

Even if a contract improves the welfare of all generations involved, generations may have an incentive to default on the provisions of the contract in the absence of legal enforcement. As legal enforcement may be difficult in the context of intergenerational contracts, we have also characterized the conditions under which a series of contracts is self-enforcing in the sense that no generation has an incentive to deviate in a non-cooperative setting. We have derived a condition which ensures the existence of a set of incentive compatible combinations of mitigation share and transfer. Finally, we have shown that for high enough income, it is possible to find combinations of the mitigation share and transfer for which a contract scheme is simultaneously Pareto improving and self-enforcing. For such combinations, institutions are still needed to provide information, coordinate payments and surveil compliance with the contract scheme. But mitigation will be beneficial for and supported by voters in each period so that political pressure is minimized.

Two strengths of our work are the general equilibrium perspective of the model and the full dynamic analysis given. Further, a contribution to existing literature is the accounting for commitment problems in a general equilibrium setup. Our model could be extended in several ways. In particular, studies on how technical change and population growth affect the results of our paper are under way. 


\section{Appendix}

\section{A1. Proof of lemma 1}

We defined the boundary of $\mathcal{S}_{t+1}^{o}$ as

$$
\underline{\mathcal{S}}_{t+1}^{o}=\left\{\left(m_{t}, \tau_{t+1}^{o}\right) \in\left[0, \min \left\{1, \bar{m}_{t}\right\}\right] \times \mathbb{R}, m_{t}<1: \Delta V_{t}^{t+1}=0\right\}
$$

i.e.

$$
\tau_{t+1}^{o}=\left(\frac{1}{1-m_{t}}\right)^{\frac{1}{\beta}+\alpha}-1 \equiv \Omega\left(m_{t}\right) \quad \text { with } \quad m_{t} \in\left[0, \min \left\{1, \bar{m}_{t}\right\}\right], m_{t}<1
$$

All pairs $\left(m_{t}, \tau_{t+1}^{o}\right) \in\left[0, \min \left\{1, \bar{m}_{t}\right\}\right] \times \mathbb{R}$ above the boundary $\underline{\mathcal{S}}_{t+1}^{o}$ belong to the set $\mathcal{S}_{t+1}^{o}$. In order to prove that $\mathcal{S}_{t+1}^{o}$ is convex, it is sufficient to prove that $\Omega\left(m_{t}\right)$ is convex in $m_{t} \in$ $\left[0, \min \left\{1, \bar{m}_{t}\right\}\right]$. This proof is straightforward from the last equation.

Similarly we defined the boundary of $\mathcal{S}_{t+1}^{y}$ as

$$
\underline{\mathcal{S}}_{t+1}^{y}=\left\{\left(m_{t}, \tau_{t+1}^{o}\right) \in\left[0, \min \left\{1, \bar{m}_{t}\right\}\right] \times \mathbb{R}, m_{t}<1: \Delta \mathcal{V}_{t+1}^{t+1}=0\right\}
$$

i.e.

$\tau_{t+1}^{o}=\frac{1-\alpha}{\alpha}\left[1-\mathrm{e}^{-\frac{(\beta+\gamma+\gamma \beta) \beta m_{t} I_{t}}{(1+\beta)(1+\alpha \beta)}}\left(1-m_{t}\right)^{-\alpha}\right] \equiv \psi\left(m_{t}, I_{t}\right) \quad$ with $\quad m_{t} \in\left[0, \min \left\{1, \bar{m}_{t}\right\}\right], m_{t}<1$

All pairs $\left(m_{t}, \tau_{t+1}^{o}\right) \in\left[0, \min \left\{1, \bar{m}_{t}\right\}\right] \times \mathbb{R}$ below the boundary $\underline{\mathcal{S}}_{t+1}^{y}$ belong to the set $\mathcal{S}_{t+1}^{y}$. So in order to prove that $\mathcal{S}_{t+1}^{y}$ is convex, it is sufficient to prove that $\psi\left(m_{t}, I_{t}\right)$ is concave in $m_{t} \in\left[0, \min \left\{1, \bar{m}_{t}\right\}\right]$. Indeed, we have

$$
\psi_{m m}\left(m_{t}, I_{t}\right)=(\alpha-1) \frac{\mathrm{e}^{-a m_{t} I_{t}}}{(1-m)^{\alpha}}\left[\frac{1}{\left(1-m_{t}\right)^{2}}+\left(\frac{a I_{t}}{\sqrt{\alpha}}-\frac{\sqrt{\alpha}}{1-m_{t}}\right)^{2}\right]<0 \quad \forall m_{t} \in[0,1)
$$

where $a=\frac{\beta(\beta+\gamma+\gamma \beta)}{\alpha(1+\beta)(1+\alpha \beta)}$.

\section{A2. Proof of proposition 1}

We see from (42) and (43) that

$$
\Omega(0)=\psi\left(0, I_{t}\right)=0
$$

Now we take into account the slopes of $\Omega\left(m_{t}\right)$ and $\psi\left(m_{t}, I_{t}\right)$ as $m_{t}$ approaches 0 from the right. We have 


$$
\Omega^{\prime}\left(0^{+}\right)=\lim _{m_{t} \rightarrow 0^{+}} \frac{1+\alpha \beta}{\beta}\left(\frac{1}{1-m_{t}}\right)^{1+\alpha+\frac{1}{\beta}}=\frac{1}{\beta}+\alpha
$$

and

$$
\begin{aligned}
\psi_{m}\left(0^{+}, I_{t}\right)=\frac{1-\alpha}{\alpha} \lim _{m_{t} \rightarrow 0^{+}} & {\left[\frac{(\beta+\gamma+\gamma \beta) \beta I_{t}}{(1+\beta)(1+\alpha \beta)}-\frac{\alpha}{1-m_{t}}\right] \mathrm{e}^{-\frac{(\beta+\gamma+\gamma \beta) \beta m_{t} I_{t}}{(1+\beta)(1+\alpha \beta)}}\left(1-m_{t}\right)^{-\alpha} } \\
= & (1-\alpha)\left[\frac{(\beta+\gamma+\gamma \beta) \beta I_{t}}{\alpha(1+\beta)(1+\alpha \beta)}-1\right]
\end{aligned}
$$

It comes from the convexity of $\Omega\left(m_{t}\right)$, the concavity of $\psi\left(m_{t}, I_{t}\right)$, and (44) that $\mathcal{P}_{t+1}^{t} \neq \varnothing$ if, and only if,

$$
\begin{gathered}
\Omega^{\prime}\left(0^{+}\right)=\frac{1}{\beta}+\alpha<(1-\alpha)\left[\frac{(\beta+\gamma+\gamma \beta) \beta I_{t}}{\alpha(1+\beta)(1+\alpha \beta)}-1\right]=\psi_{m}\left(0^{+}, I_{t}\right) \\
\Longleftrightarrow I_{t}>\frac{\alpha(1+\alpha \beta)(1+\beta)^{2}}{(1-\alpha)(\beta+\gamma+\gamma \beta) \beta^{2}}=\hat{I}
\end{gathered}
$$

\section{A3. Proof of lemma 2}

This statement is trivially true for the case $\mathcal{P}_{t+1}^{t}=\oslash$. We now just focus on the case $\mathcal{P}_{t+1}^{t} \neq \oslash$. We have

$$
\mathcal{P}_{t+1}^{t} \cup \underline{\mathcal{P}}_{t+1}^{t}=\left(\mathcal{S}_{t+1}^{o} \cup \underline{\mathcal{S}}_{t+1}^{o}\right) \cap\left(\mathcal{S}_{t+1}^{y} \cup \underline{\mathcal{S}}_{t+1}^{y}\right)
$$

Since $\mathcal{S}_{t+1}^{o} \cup \underline{\mathcal{S}}_{t+1}^{o}$ and $\mathcal{S}_{t+1}^{y} \cup \underline{\mathcal{S}}_{t+1}^{y}$ are closed sets, $\mathcal{P}_{t+1}^{t} \cup \underline{\mathcal{P}}_{t+1}^{t}$ is closed. Moreover, since $\psi\left(m_{t}, I_{t}\right)$ is continuous over $m_{t} \in[0,1)$ and $\psi\left(0, I_{t}\right)=0, \lim _{m_{t} \rightarrow 1^{-}} \psi\left(m_{t}, I_{t}\right)=-\infty$ for all $I_{t}<+\infty$, it is true that $\exists N<+\infty$ such that $\psi\left(m_{t}, I_{t}\right)<N$ for all $m_{t} \in[0,1)$ and $I_{t}<+\infty$. Thus $\mathcal{P}_{t+1}^{t} \cup \underline{\mathcal{P}}_{t+1}^{t}$ is always bounded by the ball $B_{R}(0,0)$ of center $(0,0)$ and some finite radius $R \geq \sqrt{1+N^{2}}$. Therefore, $\mathcal{P}_{t+1}^{t} \cup \underline{\mathcal{P}}_{t+1}^{t}$ is compact.

\section{A4. Proof of proposition 2}

Since both $\triangle V_{t}^{t+1}$ and $\triangle \mathcal{V}_{t+1}^{t+1}$ are well-defined and continuous over the set $\mathcal{P}_{t+1}^{t} \cup \underline{\mathcal{P}}_{t+1}^{t}$, the product $\triangle V_{t}^{t+1} \triangle \mathcal{V}_{t+1}^{t+1}$ is continuous over $\mathcal{P}_{t+1}^{t} \cup \underline{\mathcal{P}}_{t+1}^{t}$. So the existence of $\left(m_{t}^{*}, \tau_{t+1}^{o *}\right)$ is guaranteed by the compactness of $\mathcal{P}_{t+1}^{t} \cup \underline{\mathcal{P}}_{t+1}^{t}$ which has been proven in lemma 2 . It is trivial to rule out the case where at least one of the contraints $\Delta V_{t}^{t+1} \geq 0$ and $\Delta \mathcal{V}_{t+1}^{t+1} \geq 0$ is binding because in this case the objective function $\triangle V_{t}^{t+1} \triangle \mathcal{V}_{t+1}^{t+1}=0$ while any other point $\left(m_{t}, \tau_{t+1}^{o}\right) \in \mathcal{P}_{t+1}^{t}$ gives $\triangle V_{t}^{t+1} \triangle \mathcal{V}_{t+1}^{t+1}>0$. So at the maxium none of these two contraints is binding. Hence, the Lagragian for the optimization is 


$$
\mathcal{L}\left(m_{t}, \tau_{t+1}^{o}, \mu\right)=\triangle V_{t}^{t+1} \triangle \mathcal{V}_{t+1}^{t+1}+\mu\left(m_{t}-\bar{m}_{t}\right)
$$

where $\mu \geq 0$ is the Lagrangian multiplier for the constraint $m_{t}-\bar{m}_{t} \leq 0$. The first-order Kuhn-Tucker conditions at the optimal point $\left(m_{t}, \tau_{t+1}^{o}\right) \in \mathcal{P}_{t+1}^{t}$ are

$$
\begin{gathered}
\mathcal{L}_{m}\left(m_{t}^{*}, \tau_{t+1}^{o *}, \mu\right)=\mu-\frac{1+\alpha \beta}{1-m_{t}^{*}} \Delta \mathcal{V}_{t+1}^{t+1, *}+\triangle V_{t}^{t+1, *}\left[\frac{\beta+\gamma+\gamma \beta}{1+\beta} \beta I_{t}-\frac{\alpha(1+\alpha \beta)}{1-m_{t}^{*}}\right]=0 \\
\mathcal{L}_{\tau}\left(m_{t}^{*}, \tau_{t+1}^{o *}, \mu\right)=\frac{\beta}{1+\tau_{t+1}^{o *}} \Delta \mathcal{V}_{t+1}^{t+1, *}-\triangle V_{t}^{t+1, *} \frac{\alpha(1+\alpha \beta)}{1-\alpha\left(1+\tau_{t+1}^{o *}\right)}=0 \\
\mu\left(m_{t}^{*}-\bar{m}_{t}\right)=0
\end{gathered}
$$

(i) If $m_{t}^{*}=\bar{m}_{t}=\frac{(1-\delta)(1+\beta) E_{t} / I_{t}+\beta}{\beta+\gamma+\gamma \beta}$, then from (47) we have

$$
\begin{aligned}
Q\left(\tau_{t+1}^{o *}\right) \equiv & \frac{\beta}{1+\tau_{t+1}^{o *}}\left\{(1+\alpha \beta) \ln \left[\left(\frac{1-\alpha\left(1+\tau_{t+1}^{o *}\right)}{1-\alpha}\right)\left(1-\bar{m}_{t}\right)^{\alpha}\right]+\frac{\beta+\gamma+\gamma \beta}{1+\beta} \beta \bar{m}_{t} I_{t}\right\} \\
& -\left\{(1+\alpha \beta) \ln \left(1-\bar{m}_{t}\right)+\beta \ln \left(1+\tau_{t+1}^{o *}\right)\right\} \frac{\alpha(1+\alpha \beta)}{1-\alpha\left(1+\tau_{t+1}^{o *}\right)}=0
\end{aligned}
$$

where $Q\left(\tau_{t+1}^{o *}\right)$ is decreasing in $\tau_{t+1}^{o *}$, and

$$
Q(0)=\beta^{2} \frac{\beta+\gamma+\gamma \beta}{1+\beta} \bar{m}_{t} I_{t}-\frac{\alpha(1+\alpha \beta)^{2}}{1-\alpha} \ln \left(1-\bar{m}_{t}\right)>0
$$

and

$$
\lim _{\tau_{t+1}^{o *} \rightarrow\left(\frac{1-\alpha}{\alpha}\right)^{-}} Q\left(\tau_{t+1}^{o *}\right)=-\infty
$$

Hence, there always exists a unique $\tau_{t+1}^{o *} \in\left(0, \frac{1-\alpha}{\alpha}\right)$ solving (48), implying that there always exits a unique $\left(m_{t}, \tau_{t+1}^{o}\right)=\left(\bar{m}_{t}, \tau_{t+1}^{o *}\right)$ solving the Nash bargaining problem in case the contraint $m_{t} \leq \bar{m}_{t}$ is binding.

(ii) If $m_{t}^{*}<\bar{m}_{t}$, then $\mu=0$ and from (46) and (47) we find that

$$
\begin{gathered}
\frac{1+\tau_{t+1}^{o *}}{1-m_{t}^{*}} \frac{1+\alpha \beta}{\beta}=\left[\beta \frac{\beta+\gamma+\gamma \beta}{1+\beta} I_{t}-\frac{\alpha(1+\alpha \beta)}{1-m_{t}^{*}}\right] \frac{1-\alpha\left(1+\tau_{t+1}^{o *}\right)}{\alpha(1+\alpha \beta)} \\
\Longrightarrow 1+\tau_{t+1}^{o *}=\frac{\beta^{2} \frac{\beta+\gamma+\gamma \beta}{1+\beta} I_{t}\left(1-m_{t}^{*}\right)-\alpha \beta(1+\alpha \beta)}{\alpha\left(\beta^{2} \frac{\beta+\gamma+\gamma \beta}{1+\beta} I_{t}\left(1-m_{t}^{*}\right)+1+\alpha \beta\right)}
\end{gathered}
$$


It can be shown that equation (49) denotes the Pareto frontier. Since $\tau_{t+1}^{o *}>0$, it follows from (49) that

$$
1-m_{t}^{*}>\frac{\alpha(1+\alpha \beta)(1+\beta)^{2}}{(1-\alpha)(\beta+\gamma+\gamma \beta) \beta^{2} I_{t}} \quad \text { i.e. } \quad m_{t}^{*}<1-\frac{\alpha(1+\alpha \beta)(1+\beta)^{2}}{(1-\alpha)(\beta+\gamma+\gamma \beta) \beta^{2} I_{t}}=\hat{m}_{t}
$$

Substituting (49) into (46), we find $m_{t}^{*}$ as the solution to

$$
\begin{aligned}
\hat{Q}\left(m_{t} ; I_{t}\right)=\ln & {\left[\left(1-m_{t}\right)^{1+\alpha \beta}\left(\frac{\beta b I_{t}\left(1-m_{t}\right)-\alpha \beta(1+\alpha \beta)}{\alpha\left(\beta b I_{t}\left(1-m_{t}\right)+1+\alpha \beta\right)}\right)^{\beta}\right]^{b I_{t}-\frac{\alpha(1+\alpha \beta)}{1-m}} } \\
& -\frac{1+\alpha \beta}{1-m_{t}}\left\{\ln \left[\frac{\left(1-\alpha^{2} \beta^{2}\right)\left(1-m_{t}\right)^{\alpha}}{(1-\alpha)\left(\beta b I_{t}\left(1-m_{t}\right)+1+\alpha \beta\right)}\right]^{1+\alpha \beta}+b m_{t} I_{t}\right\}=0
\end{aligned}
$$

where $b=\frac{\beta(\beta+\gamma+\gamma \beta)}{1+\beta}$.

The existence of a solution to (51) is proven by the existence of a solution $\left(m_{t}, \tau_{t+1}^{o}\right)$ to the Nash bargaining problem. The uniqueness of $m_{t}^{*}$ is guaranteed by the monotonicity of the function $\hat{Q}\left(m_{t}^{*} ; I_{t}\right)$. We now prove that for all $I_{t}>\hat{I}, \hat{Q}\left(m_{t}^{*} ; I_{t}\right)$ is decreasing in $m_{t}^{*}$. From (51), it is sufficient to prove that $\frac{\left(1-m_{t}\right)^{\alpha}}{\beta b I_{t}\left(1-m_{t}\right)+1+\alpha \beta}$ is increasing in $m_{t}$ for all $I_{t}>\hat{I}$ and $m_{t}<\hat{m}_{t}$. In effect, its derivative with respect to $m_{t}$ is

$$
\frac{(1-\alpha)\left(1-m_{t}\right) \beta b I_{t}-\alpha(1+\alpha \beta)}{\left(1-m_{t}\right)^{1-\alpha}\left[\beta b I_{t}\left(1-m_{t}\right)+1+\alpha \beta\right]^{2}}>0 \quad \forall m_{t}<\hat{m}_{t}, \forall I_{t}>\hat{I}
$$

Therefore, there exists a unique $m_{t}^{*}=m\left(I_{t}\right) \in\left(0, \hat{m}_{t}\right)$ that solves (51), i.e., in this case there exists a unique interior pair $\left(m_{t}^{*}, \tau_{t+1}^{o *}\right) \equiv\left(m\left(I_{t}\right), \tau\left(I_{t}\right)\right) \in \mathcal{P}_{t+1}^{t}$ that solves the Nash bargaining problem. Since $\left(m_{t}^{*}, \tau_{t+1}^{o *}\right)$ is the unique pair solving the first order conditions (46) and (47), it is the unique stationary point of the function $\triangle V_{t}^{t+1} \triangle \mathcal{V}_{t+1}^{t+1}$ defined over the set $\mathcal{P}_{t+1}^{t} \cup \underline{\mathcal{P}}_{t+1}^{t}$.

\section{A5. Proof of lemma 3}

Since $m(\hat{I})=0, m(\bar{I})>\frac{\beta}{\beta+\gamma+\gamma \beta}$ and $m^{\prime}\left(I_{t}\right)>0$ for all $I_{t} \in(\hat{I}, \bar{I})$ then there exists a unique $\underline{I} \in(\hat{I}, \bar{I})$ such that $m(\underline{I})=\frac{\beta}{\beta+\gamma+\gamma \beta}$. From (30) we have

$$
m\left(I_{t}\right)-\frac{(1-\delta)(1+\beta) E_{t}+\beta I_{t}}{(\beta+\gamma+\gamma \beta) I_{t}}=0
$$

implies for all $I_{t} \in[\underline{I}, \bar{I})$, there exists a unique $\hat{E}_{t}$ solving (52), and

$$
\hat{E}_{t}=\frac{(\beta+\gamma+\gamma \beta) m\left(I_{t}\right)-\beta}{(1-\delta)(1+\beta)} I_{t} \equiv \hat{E}\left(I_{t}\right)
$$


where $\hat{E}(\underline{I})=0$ and $\hat{E}^{\prime}\left(I_{t}\right)>0$ for all $I_{t} \in[\underline{I}, \bar{I}]$.

\section{A6. Proof of proposition 3}

Under the dynamic system (31)-(32), the steady state is characterized by the following function

$$
\varphi(E) \equiv E-\frac{1}{\delta}\left[\frac{\beta(1-\alpha)}{1+\beta} A \mathrm{e}^{-E}\right]^{\frac{1}{1-\alpha}}=0
$$

We have

$$
\varphi^{\prime}(E)=1+\frac{1}{(1-\alpha) \delta}\left[\frac{\beta(1-\alpha)}{1+\beta} A \mathrm{e}^{-E}\right]^{\frac{1}{1-\alpha}}>0
$$

and

$$
\varphi(0)=-\frac{1}{\delta}\left[\frac{\beta(1-\alpha)}{1+\beta}\right]^{\frac{1}{1-\alpha}}<0 \quad \text { and } \quad \lim _{E \rightarrow+\infty} \varphi(E)=+\infty
$$

Hence, there exists a unique steady state which is characterized by (53). The steady state income of the agent in this case is

$$
\tilde{I}=A^{\frac{1}{1-\alpha}} \mathrm{e}^{-\frac{\tilde{E}}{1-\alpha}}\left(\frac{\beta}{1+\beta}\right)^{\frac{\alpha}{1-\alpha}}(1-\alpha)^{\frac{1}{1-\alpha}}
$$

The Jacobian matrix associated with the dynamic system (31)-(32) evaluated at the steady state $(\tilde{I}, \tilde{E})$ is as follows:

$$
\tilde{J}=\left(\begin{array}{cc}
\alpha & -\frac{1+\beta}{\beta} \delta \tilde{E} \\
\frac{\beta}{1+\beta} & 1-\delta
\end{array}\right)
$$

Its determinant and trace are

$$
\operatorname{det}(\tilde{J})=\alpha(1-\delta)+\delta \tilde{E}>0 ; \quad \operatorname{Tr}(\tilde{J})=\alpha+1-\delta>0
$$

and the characteristic function is

$$
C(\lambda)=\lambda^{2}-\operatorname{Tr}(\tilde{J}) \lambda+\operatorname{det}(\tilde{J})
$$

(i) If $\operatorname{Tr}(\tilde{J})^{2}>4 \operatorname{det}(\tilde{J})$, there are two distinct real eigenvalues $\lambda_{1}, \lambda_{2}$. We have

$$
C(-1)=1+\operatorname{Tr}(\tilde{J})+\operatorname{det}(\tilde{J})>C(1)=\delta(1-\alpha)+\delta \tilde{E}>0
$$

Hence, in this case we have two distinct eigenvalues $\lambda_{1}, \lambda_{2} \in(0,1)$. The steady state $(\tilde{I}, \tilde{E})$ 
is a stable node.

(ii) If $\operatorname{Tr}(\tilde{J})^{2}=4 \operatorname{det}(\tilde{J})$, we have a pair of repeated real eigenvalues $\lambda=\frac{\alpha+1-\delta}{2} \in(0,1)$. The steady state $(\tilde{I}, \tilde{E})$ is stable.

(iii) If $\operatorname{Tr}(\tilde{J})^{2}<4 \operatorname{det}(\tilde{J})$, we have two complex eigenvalues. It is obvious that

$$
\varphi\left(\frac{1-\alpha(1-\delta)}{\delta}\right)>0 \Longrightarrow \tilde{E}<\frac{1-\alpha(1-\delta)}{\delta}
$$

Hence

$$
\operatorname{det}(\tilde{J})=\alpha(1-\delta)+\delta \tilde{E}<1
$$

Therefore, in this case, the steady state $(\tilde{I}, \tilde{E})$ is a spiral sink.

\section{A7. Proof of proposition 5}

According to lemma 6 , a sustainable path with $m_{t}>0, \tau_{t+1}^{o}>0$ for every $t=T \ldots \infty$ exists if and only if the stationary set $S^{I C}$ is non-empty, $S^{I C} \neq \oslash$, with $S^{I C}$ given by (38).

As can be seen from (39), the function $m^{I C}\left(\tau^{o}\right)$ delineating the boundary of $S^{I C}$ is concave, with $m^{I C}(0)=0$. $S^{I C} \neq \oslash$ if and only if the slope of the IC-curve at the origin is positive. Formally, this is expressed by the condition $\frac{\partial m^{I C}}{\partial \tau^{o}}(0)=\frac{\beta-\frac{\alpha}{1-\alpha}(1+\alpha \beta)}{1+\alpha \beta}>0$ which yields condition (40). If condition (40) does not hold, only negative values of $\tau^{o}$ satisfy the incentive constraint. To see that (40) is also sufficient, note that the IC-curve is continuous in $\tau^{o}$ for $\tau^{o} \epsilon\left[0, \tau^{o, \max }\right)$. A sustainable equilibrium path with positive transfer payments and positive mitigation investment in each period will therefore exist under condition (40) for sufficiently small mitigation levels $m_{t}$. Under condition (40), $m^{I C}=0$ not only at the origin but also for some positive $\bar{\tau}^{o}<\tau^{o, \max }$ and $\lim _{\tau^{o} \rightarrow \tau^{o, \max }} m^{I C}\left(\tau^{o}\right)=-\infty$. Hence, a positive mitigation share $m>0$ can only be sustained for $0<\tau^{o}<\bar{\tau}^{o}$.

The existence of a maximum sustainable mitigation share $m$ follows from the shape of the function $m^{I C}\left(\tau^{o}\right)$, which is increasing for small but decreasing for large values of $\tau^{o}$. The maximum sustainable $m$ is derived by setting $\frac{\partial m^{I C}}{\partial \tau^{o}}=0$. Solving for $\tau^{o}$ and substituting the solution back into (39), we obtain:

$$
m^{\max }=1-\left(\frac{\alpha}{1-\alpha} \frac{1+\alpha \beta}{\beta}\right)^{\frac{1+\alpha \beta}{\beta}}\left((1-\alpha) \frac{\beta+1+\alpha \beta}{1+\alpha \beta}\right)^{1+\frac{1+\alpha \beta}{\beta}}<1
$$

$m^{\max }$ is strictly smaller than one.

\section{A8. Heuristic derivation of condition (40)}

Condition (40) can be derived by explicitly comparing returns from pure capital investment and capital investment combined with the transfer scheme: Assume that there is no social 
contract, i.e. $m_{t}=\tau_{t}^{o}=\tau_{t+1}^{o}=0$. Marginally increasing the transfer $\tau_{t}^{o}$ to the current old generation yields a perfectly foreseen return of $R_{t+1}^{e} k_{t+1} d \tau_{t+1}^{o, e}=R_{t+1} k_{t+1} d \tau_{t+1}^{o}$ in period $t+1$. Further, an agent of generation $t$ takes into account the equilibrium effect of slower capital accumulation due to lower income on the perfectly foreseen return to capital, $\frac{\partial R_{t+1}}{\partial k_{t+1}} \frac{\partial k_{t+1}}{\partial \tau_{t}^{o}} d \tau_{t}^{o}>0$, which is associated with a total change in return of $\frac{\partial R_{t+1}}{\partial k_{t+1}} \frac{\partial k_{t+1}}{\partial \tau_{t}^{o}} d \tau_{t}^{o} k_{t+1}=(1-\alpha) \frac{\beta}{1+\beta} R_{t+1} R_{t} k_{t} d \tau_{t}^{o}$. On the other hand, increasing the transfer $\tau_{t}^{o}$ reduces income in period $t$ by $R_{t} k_{t} d \tau_{t}^{o}$. Investing this income in physical capital instead would yield an expected return of $R_{t+1} R_{t} k_{t} d \tau_{t}^{o}$ (as $\tau_{t+1}^{o, e}=0$ without social contract). The agent will prefer to combine capital investment with investing in the transfer system and the social contract over investing only in capital if and only if

$$
R_{t+1} k_{t+1} d \tau_{t+1}^{o}+(1-\alpha) \frac{\beta}{1+\beta} R_{t+1} R_{t} k_{t} d \tau_{t}^{o}-R_{t+1} R_{t} k_{t} d \tau_{t}^{o}>0
$$

Assuming a stationary transfer system, so that $d \tau_{t}^{o}=d \tau_{t+1}^{o}$, this condition becomes

$$
R_{t+1} k_{t+1}+(1-\alpha) \frac{\beta}{1+\beta} R_{t+1} R_{t} k_{t}-R_{t+1} R_{t} k_{t}>0
$$

After some simplification, taking into account $\tau_{t}^{y}=0$, so that $R_{t}=\alpha z\left(E_{t-1}\right) k_{t}^{\alpha-1}$ and $k_{t+1}=$ $\frac{\beta}{1+\beta} z\left(E_{t-1}\right)(1-\alpha) k_{t}^{\alpha}$, the condition is equivalent to

$$
\begin{aligned}
1-\frac{\alpha}{(1-\alpha) \frac{\beta}{1+\beta}}\left(1-(1-\alpha) \frac{\beta}{1+\beta}\right) & >0 \\
\Leftrightarrow \beta & >\frac{\alpha}{1-\alpha}(1+\alpha \beta)
\end{aligned}
$$

which is condition (40).

\section{A9. Proof of proposition 6}

Fulfillment of condition (41) in period $T$ is necessary and sufficient for the set $S^{I C} \cap \mathcal{P}_{T+1}^{T}$ to be non-empty in the starting period of the contract scheme. If the condition holds also for every $t>T$, then there exists some pair $\left(m, \tau^{o}\right), m>0, \tau^{o}>0$ in the set $S^{I C} \cap \mathcal{P}_{T+1}^{T}$ which will also lie in every set $S^{I C} \cap \mathcal{P}_{t+1}^{t}$ for $t>T$. Such a pair can be maintained as incentive compatible, Pareto improving contract for $t \rightarrow \infty$.

Condition (41) is derived as follows: The boundary of the set $S^{I C}$ is the stationary incentive constraint $m^{I C}\left(\tau^{o}\right)$ in (39), while the boundaries of the Pareto improvement set $\mathcal{P}_{t+1}^{t}$ are given by $\psi\left(m_{t} ; I_{t}\right)$ in $(43)$ and $\Omega\left(m_{t}\right)$ in $(42)$. Because the stationary incentive constraint is defined as $m^{I C}\left(\tau^{o}\right)$ while the boundaries of the Pareto improvement set are defined as functions of $m$, we first invert the function $m^{I C}\left(\tau^{o}\right)$ over the intervall $\tau^{o} \epsilon\left[0, \tau^{o}\left(m^{\max }\right)\right]$, where $m^{\max }$ is defined in (54). Denote the inverse as $\tau^{o, I C}(m)$.

In $\left(m_{t}, \tau_{t+1}^{o}\right)$-space, $\tau^{o, I C}(m)$ lies above the lower boundary $\Omega\left(m_{t}\right)$ of the Pareto improve- 
ment area for $m_{t}, \tau_{t+1}^{o}>0, \forall t . \quad \tau^{o, I C}(m)$ and the upper boundary $\psi\left(m_{t} ; I_{t}\right)$ of the Pareto improvement area intersect at the origin $\left(m_{t}, \tau_{t+1}^{o}\right)=(0,0)$ for all $t$. Given the functional forms of the two curves, they enclose a non-empty set of pairs $\left(m_{t}, \tau_{t+1}^{o}\right), m_{t}, \tau_{t+1}^{o}>0$ (so that $\left.S^{I C} \cap \mathcal{P}_{t+1}^{t} \neq \oslash\right)$ in a period $t$ if and only if at the origin, the slope of $\tau^{o, I C}(m)$ is flatter than the slope of $\psi\left(m_{t} ; I_{t}\right)$, i.e. $\tau_{m}^{o, I C}(0)<\psi_{m}\left(0^{+}, I_{t}^{g}\right)$.

From (39), it follows that the derivative $\tau_{m}^{o, I C}(0)$ is:

$$
\tau_{m}^{o, I C}(0)=\frac{1+\alpha \beta}{\beta-\frac{\alpha}{1-\alpha}(1+\alpha \beta)}
$$

The numerator gives the absolute value of the marginal utility loss from an increase in the mitigation share $m$ from zero. The denominator contains the marginal utility gain from an increase in $\tau^{o}$ net of the marginal utility loss incurred by an increase in the transfer paid, $\tau^{y}$. Under condition (40), the denominator and therefore $\tau_{m}^{o, I C}(0)$ is strictly positive.

The slope of the upper boundary $\psi\left(m_{t} ; I_{t}\right)$ at the origin was shown to be

$$
\psi_{m}\left(0^{+}, I_{t}\right)=(1-\alpha)\left(\frac{\beta+\gamma(1+\beta)}{\alpha(1+\beta)(1+\alpha \beta)} \beta I_{t}-1\right)
$$

which, setting $\tau_{t}^{o}=0$, becomes $\psi_{m}\left(0^{+}, I_{t}^{g}\right)=(1-\alpha)\left(\frac{\beta+\gamma(1+\beta)}{\alpha(1+\beta)(1+\alpha \beta)} \beta I_{t}^{g}-1\right)$.

The relation

$$
\tau_{m}^{o, I C}(0)<\psi_{m}\left(0^{+}, I_{t}^{g}\right)
$$

is satisfied if and only if

$$
\begin{gathered}
\frac{1+\alpha \beta}{\beta-\frac{\alpha}{1-\alpha}(1+\alpha \beta)}<(1-\alpha)\left(\frac{\beta+\gamma(1+\beta)}{\alpha(1+\beta)(1+\alpha \beta)} \beta I_{t}^{g}-1\right) \\
\Longleftrightarrow I_{t}^{g}>\frac{(1-\alpha) \frac{\beta}{1+\beta}(1+\alpha \beta+\beta)}{\beta-\frac{\alpha}{1-\alpha}(1+\alpha \beta)} \underbrace{\frac{\alpha(1+\beta)^{2}(1+\alpha \beta)}{(1-\alpha) \beta^{2}(\beta+\gamma(1+\beta))}}_{\widehat{I}_{t}}>\widehat{I}_{t}
\end{gathered}
$$

which is condition (41). The new threshold is larger than $\widehat{I}_{t}$. 


\section{References}

Anderberg, D., And Balestrino, A. Self-enforcing intergenerational transfer and the provision of education. Economica 70 (2003), 55-71.

Boldrin, M., And Rustichini, A. Political equilibria with social security. Review of Economic Dynamics 3 (2000), 41-78.

Bovenberg, L. A., and Heijdra, B. J. Environmental tax policy and intergenerational redistribution. Journal of Public Economics 67 (1998), 1-24.

Cigno, A. Intergenerational transfers without altruism: family, market and state. European Journal of Political Economy 9, 4 (1993), 505-518.

Cigno, A. A constitutional theory of the family. Journal of Population Economics 19, 2 (2006), 259-283.

Cigno, A. The political economy of intergenerational cooperation. Handbook of the economics of giving, altruism and reciprocity 2 (2006), 1505-1558.

DaO, N. T., AND DÁVILA, J. Implementing steady state efficiency in overlapping generations economies with environmental externalities. Journal of public economic theory 16, 4 (2014), 620-649.

Dao, N. T., And Edenhofer, O. On the fiscal strategies of escaping poverty-environment traps (and) towards sustainable growth. CESifo Working Paper No. 48654 (June 2014).

Fodha, M., And Seegmuller, T. Environmental quality, public debt and economic development. Environmental and Resource Economics 57, 4 (2014), 487-504.

Foley, D. K. The economic fundamentals of global warming. New York, Department of Economics, New School for Social Research, October 2007.

Gerlagh, R., and Keyzer, M. A. Sustainability and the intergenerational redistribution of natural resource entitlements. Journal of Public Economics 79 (2001), 315-341.

Goenka, A., Jafarey, S., Pouliot, W., et al. Pollution, mortality and optimal environmental policy. Tech. rep., 2012.

Gutiérrez, M.-J. Dynamic inefficiency in an overlapping generation economy with pollution and health costs. Journal of Public Economic Theory 10, 4 (2008), 563-594.

Howarth, R. B., and Norgand, R. B. Environmental evaluation under sustainable development. The American Economic Review 82, 2 (1992), 473-477.

John, A., And Pecchenino, R. An overlapping generations model of growth and the environment. The Economic Journal (1994), 1393-1410.

Jouvet, P.-A., Michel, P., And Vidal, J.-P. Intergenerational altruism and the environment. The Scandinavian Journal of Economics 102, 1 (2000), 135-150.

Karp, L., And ReZai, A. The political economy of environmental policy with overlapping generations. International Economic Review 55, 3 (2014), 711-733.

NASH JR, J. F. The bargaining problem. Econometrica: Journal of the Econometric Society (1950), 155-162. 
Ono, T. Optimal tax schemes and the environmental externality. Economics Letters 53, 3 (1996), 283-289.

RANGEL, A. Forward and backward intergenerational goods: Why is social security good for the environment? The American Economic Review 93, 3 (2003).

von Below, D., Dennig, F., And JaAkkola, N. Consuming more and polluting less today: Intergenerationally efficient climate policy. mimeo, 2015 . 